\title{
Lepton flavor violation in the inert scalar model with higher representations
}

\author{
Talal Ahmed Chowdhury ${ }^{a}$ and Salah Nasri ${ }^{b}$ \\ ${ }^{a}$ Department of Physics, University of Dhaka, \\ P.O. Box 1000, Dhaka, Bangladesh \\ ${ }^{b}$ Department of Physics, UAE University, \\ P.O. Box 17551, Al-Ain, United Arab Emirates \\ E-mail: talal@du.ac.bd, snasri@uaeu.ac.ae
}

\begin{abstract}
We investigate the lepton flavor violation (LFV) in the inert scalar model with higher representations. We generalize the inert doublet model with right handed neutrino by using higher scalar and fermion representation of $\mathrm{SU}(2)_{L}$. As the generalized model and the inert doublet model have the same parameter space, we compare the rates of $\mu \rightarrow e \gamma$, $\mu \rightarrow e e \bar{e}$ and $\mu-e$ conversion in nuclei in the doublet and its immediate extension, the quartet model. We show that the corresponding rates are larger in the case of higher representation compared to the Inert doublet for the same region of parameter space. This implies that such extended models are more constrained by current LFV bounds and will have better prospects in future experiments.
\end{abstract}

Keywords: Rare Decays, Beyond Standard Model, Neutrino Physics

ARXIV EPRINT: 1506.00261 


\section{Contents}

1 Introduction $\quad 2$

2 The model 3

2.1 Mass spectra 4

2.2 Neutrino mass generation 5

$\begin{array}{lll}2.3 \text { Perturbativity } & 7\end{array}$

$\begin{array}{lll}3 & \text { Lepton flavor violating processes } & 7\end{array}$

$3.1 \mu \rightarrow e \gamma \quad 7$

$3.2 \mu \rightarrow e e \bar{e} \quad 8$

3.2.1 $\gamma$-penguin contribution $\quad 8$

3.2.2 Z-penguin contribution $\quad 9$

$\begin{array}{ll}3.2 .3 \text { Box contribution } & 10\end{array}$

$3.3 \mu-e$ conversion in nuclei 11

4 Results and discussion $\quad 12$

$\begin{array}{ll}4.1 \text { Constraints and parameter space } & 12\end{array}$

$\begin{array}{lll}\text { 4.1.1 Collider constraints } & 12\end{array}$

4.1.2 DM constraints 13

4.1.3 Gamma ray constraints and Sommerfeld enhancement 14

4.1.4 Scalar coupling and LFV rates with scalar DM 16

$\begin{array}{ll}\text { 4.1.5 Viable parameter space } & 17\end{array}$

$\begin{array}{lll}4.2 & \text { LFV processes } & 18\end{array}$

$\begin{array}{lll}\text { 4.2.1 } & \operatorname{Br}(\mu \rightarrow e \gamma) & 19\end{array}$

$\begin{array}{lll}4.2 .2 & \operatorname{Br}(\mu \rightarrow e e \bar{e}) & 19\end{array}$

$\begin{array}{lll}4.2 .3 & \mu-e \text { conversion rate } & 20\end{array}$

4.2.4 LFV rates in the doublet and quartet 20

5 Conclusions 21

A Scalar masses $\quad 22$

A.1 Inert doublet 22

$\begin{array}{lll}\text { A.2 Inert quartet } & 23\end{array}$

B Loop functions $\quad 23$

C $\mu e \gamma$ vertex, $\mu e Z$ vertex and box diagrams $\quad 24$

C.1

$\begin{array}{lll}\text { C.2 } & \mu e Z \text { vertex } & 25\end{array}$

$\begin{array}{lll}\text { C.3 Box diagrams } & 26\end{array}$ 


\section{Introduction}

Neutrino oscillation provides the direct evidence for lepton flavor violation in the neutrino sector. Therefore, one also expects LFV in the charged lepton sector which is yet to be observed. This is a generic prediction in most of the neutrino mass models and depending on the realization details of the model, the rates of different LFV processes can be very different. In this paper, we have focused on radiative neutrino mass model at one loop proposed in [1], known as the scotogenic model, where the scalar content of the model is the inert doublet. Apart from its role in neutrino mass generation, the inert doublet has been extensively studied in the context of dark matter [2-9], mirror model and extra generation $[10,11]$, electroweak phase transition [12-16] and collider studies [17, 18, 21]. As the higher scalar representation is not forbidden by any symmetry in the model, the immediate generalization of the doublet, the quartet with isospin $J=3 / 2$ was studied in [22] to check whether it is viable in providing both light scalar dark matter and strong electroweak phase transition in the universe. Here we have incorporated higher scalar representation instead of the doublet in the scotogenic model and determined the viable $\mathrm{SU}(2)_{L}$ fermion multiplet for generating neutrino mass. LFV processes in the scotogenic model with inert doublet has been studied in [23-28] (and references therein). The extension of the scotogenic model has been addressed in $[29,30]$. Also larger multiplets have been incorporated in type III seesaw model [31] and in models of radiative neutrino mass generation at higher order with dark matter [32].

The generalization of scotogenic model with higher $\mathrm{SU}(2)_{L}$ half-integer representation does not change the parameter set of the Lagrangian of the inert doublet at the renormalizable level. Therefore it gives us the opportunity to investigate the predictions of LFV processes for different scalar representations for the same region of parameter space. In particular, we compare the LFV processes for the doublet and the quartet in the light of current experimental bounds and future sensitivities.

There have been many great experimental efforts to detect positive LFV signal in $l_{\alpha} \rightarrow l_{\beta} \gamma, l_{\alpha} \rightarrow 3 l_{\beta}$ and $\mu-e$ conversion rate in nuclei. In the case of muon radiative decay, the MEG collaboration [33] has put a limit of $\operatorname{Br}(\mu \rightarrow e \gamma)<5.7 \times 10^{-13}$ [34] and will have sensitivity of $6 \times 10^{-14}$ after acquiring data for three more years [35]. In addition, current bound on branching ratio of lepton flavor violating 3-body decay, $\mu \rightarrow e e \bar{e}$ is $1 \times 10^{-12}$ set by SINDRUM experiment [36] and Mu3e experiment will reach a sensitivity of $10^{-16}$ [37]. Furthermore, SINDRUM II experiment has put current limit on muon to electron $(\mu-e)$ conversion rate in Gold $(\mathrm{Au})$ and Titanium (Ti) nucleus of $7 \times 10^{-13}[38]$ and $4.3 \times 10^{-12}$ [39] respectively. The future projects Mu2e [40, 41], DeeMe [42], COMET [43] and PRISM/PRIME $[44,45]$ will improve this bound from $10^{-14}$ to $10^{-18}$. For other LFV processes and their experimental bounds, please see table I of [27]. We have compared the predictions of the LFV processes $\mu \rightarrow e \gamma, \mu \rightarrow e e \bar{e}$ and $\mu-e$ conversion rate in $\mathrm{Au}$ and $\mathrm{Ti}$ for both doublet and quartet scalars and our comparison has revealed that the contributions of the quartet in all LFV processes are larger than those of the doublet for the same region of parameter space. Consequently, the contribution of higher scalar representation to LFV processes have better experimental prospects. 
The paper is organized as follows. We describe the model in section 2. In section 3 we present the relevant formulas of $\mu \rightarrow e \gamma, \mu \rightarrow e e \bar{e}$ and $\mu-e$ conversion processes for the inert doubler and quartet. We present the result in section 4 and conclude in section 5. Appendix A contains the mass spectrum of the inert doublet and quartet in our parametrization. The expressions of the loop functions are given in appendix B. In appendix $\mathrm{C}$ we collect the Feynman diagrams for $\mu e \gamma$ vertices, $\mu e Z$ vertices and box diagrams.

\section{The model}

Any multiplet charged under $\mathrm{SU}(2)_{L} \times \mathrm{U}(1)_{Y}$ gauge group is characterized by the quantum numbers $J$ and $Y$, with the electric charge of a component in the multiplet is given by $Q=T_{3}+Y$. For half-integer representation $J=n / 2, T_{3}$ ranges from $-\frac{n}{2}$ to $\frac{n}{2}$. So the hypercharge of the multiplet needs to be $Y= \pm T_{3}$ for one of the components to have neutral charge. For integer representation $n$, similar condition holds for hypercharge.

The generalized scotogenic model involves one half-integer $\mathrm{SU}(2)_{L}$ scalar multiplet $\Delta$ with hypercharge $Y=1 / 2$ and three generations of real $(Y=0)$ odd dimensional fermionic multiplets, $F_{i}(i=1-3)$ charged under $Z_{2}$ symmetry, $\Delta \rightarrow-\Delta$ and $F_{i} \rightarrow-F_{i}$. When the scalar multiplet is fixed to be $J=n / 2, n$ odd, there are two choices for fermionic multiplet which can give $Z_{2}$ even $\mathrm{SU}(2)_{L} \times \mathrm{U}(1)_{Y}$ invariant Yukawa term with the lepton doublet; $J=\frac{n-1}{2}$ or $\frac{n+1}{2}$. The charged lepton sector is augmented by the following terms

$$
\mathcal{L} \supset-\frac{M_{F_{i}}}{2} \overline{F_{i}^{c}} P_{R} F_{i}+y_{i \alpha} \bar{F}_{i} \cdot l_{\alpha} \cdot \Delta+\text { h.c }
$$

where the dot represents the proper contractions among $\mathrm{SU}(2)$ indices. In the subsequent analysis we have chosen fermion multiplet to be $J=\frac{n-1}{2}$.

The general Higgs-scalar multiplet potential, symmetric under $Z_{2}$, can be written in the following form,

$$
\begin{aligned}
V_{0}(\Phi, \Delta)= & -\mu^{2} \Phi^{\dagger} \Phi+M_{0}^{2} \Delta^{\dagger} \Delta+\lambda_{1}\left(\Phi^{\dagger} \Phi\right)^{2}+\lambda_{2}\left(\Delta^{\dagger} \Delta\right)^{2}+\lambda_{3}\left|\Delta^{\dagger} T^{a} \Delta\right|^{2}+\alpha \Phi^{\dagger} \Phi \Delta^{\dagger} \Delta \\
& +\beta \Phi^{\dagger} \tau^{a} \Phi \Delta^{\dagger} T^{a} \Delta+\gamma\left[\left(\Phi^{T} \epsilon \tau^{a} \Phi\right)\left(\Delta^{T} C T^{a} \Delta\right)^{\dagger}+h . c\right]
\end{aligned}
$$

Here, $\tau^{a}$ and $T^{a}$ are the $\mathrm{SU}(2)$ generators in fundamental and $\Delta$ 's representation respectively. $C$ is an antisymmetric matrix analogous to charge conjugation matrix defined as,

$$
C T^{a} C^{-1}=-T^{a T}
$$

Since $C$, is an antisymmetric matrix, it can only be defined for even dimensional space, i.e only for half-integer representation. If the isospin of the representation is $J$ then $C$ is $(2 J+1) \times(2 J+1)$ dimensional matrix. The generators are normalized in such a way so that they satisfy, for fundamental representation, $\operatorname{Tr}\left[\tau^{a} \tau^{b}\right]=\frac{1}{2} \delta^{a b}$ and for other representations, $\operatorname{Tr}\left(T^{a} T^{b}\right)=D_{2}(\Delta) \delta^{a b}$. Also $T^{a} T^{a}=C_{2}(\Delta)$. Here, $D_{2}(\Delta)$ and $C_{2}(\Delta)$ are Dynkin index and second Casimir invariant for $\Delta$ 's representation. Notice that, $\gamma$ term is only allowed for representation with $(J, Y)=\left(\frac{n}{2}, \frac{1}{2}\right)$ and it is essential for the generation of neutrino mass at one-loop. 
The scalar representation with $(J, Y)=\left(\frac{n}{2}, \frac{1}{2}\right)$ and the fermionic representation with $(J, Y)=\left(\frac{n-1}{2}, 0\right)$ have the component fields denoted as $\Delta^{(Q)}$ and $F^{(Q)}$ respectively where $Q$ is the electric charge. They are written explicitly as

$$
\Delta_{\frac{\mathbf{n}}{2}}=\left(\begin{array}{c}
\Delta^{\left(\frac{n+1}{2}\right)} \\
\cdots \\
\Delta^{(0)} \equiv \frac{1}{\sqrt{2}}(S+i A) \\
\cdots \\
\Delta^{\left(-\frac{n-1}{2}\right)}
\end{array}\right) \text { and } \mathbf{F}_{\frac{\mathrm{n}-1}{2}}=\left(\begin{array}{c}
F^{\left(\frac{n-1}{2}\right)} \\
\cdots \\
F^{(0)} \\
\ldots \\
F^{\left(\frac{-n+1}{2}\right)}
\end{array}\right)
$$

For the former representation every component represents a unique field while for the latter there is a redundancy $F^{(-Q)}=\left(F^{(Q)}\right)^{*}$.

The choices for real fermion multiplet with the doublet are either $(J, Y)=(0,0)$ or $(1,0)$ and with the quartet, choices are either $(J, Y)=(1,0)$ or $(2,0)$. Our analysis has focused on the following pairs of scalar and fermionic multiplets: $\left(\Delta_{J=\frac{1}{2}}, F_{i J=0}\right)$ and $\left(\Delta_{J=\frac{3}{2}}, F_{i J=1}\right)$. In component fields, the doublet scalar $D$, right handed (RH) neutrino, $N_{R_{i}}$ and the quartet scalar $\Delta$ and the triplet fermion $\mathbf{F}_{i}$ are expressed as

$$
D=\left(\begin{array}{c}
C^{+} \\
D^{0} \equiv \frac{1}{\sqrt{2}}(S+i A)
\end{array}\right), \quad N_{R_{i}}, \Delta=\left(\begin{array}{c}
\Delta^{++} \\
\Delta^{+} \\
\Delta^{0} \equiv \frac{1}{\sqrt{2}}(S+i A) \\
\Delta^{\prime}-
\end{array}\right) \text { and } \mathbf{F}_{i}=\left(\begin{array}{c}
F^{+} \\
F^{0} \\
F^{-}
\end{array}\right)_{i}
$$

\subsection{Mass spectra}

We now sketch the general form of mass spectrum for the scalar and fermionic multiplet which was also presented in [22]. The neutral component of the scalar multiplet $(Y=1 / 2)$ will have $T_{3}$ eigenvalue as $T_{3}=-\frac{1}{2}$. Now for the Higgs vacuum expectation value, $\langle\Phi\rangle=$ $\left(0, \frac{v}{\sqrt{2}}\right)^{T}$, the term $\left\langle\Phi^{\dagger}\right\rangle \tau^{3}\langle\Phi\rangle$ gives $-\frac{v^{2}}{4}$. So masses for the neutral components, $S$ and $A$ are splitted by the $\gamma$ term as

$$
\begin{aligned}
& m_{S}^{2}=M_{0}^{2}+\frac{1}{2}\left(\alpha+\frac{1}{4} \beta+p(-1)^{p+1} \gamma\right) v^{2} \\
& m_{A}^{2}=M_{0}^{2}+\frac{1}{2}\left(\alpha+\frac{1}{4} \beta-p(-1)^{p+1} \gamma\right) v^{2}
\end{aligned}
$$

Here, $p=\frac{1}{2} \operatorname{Dim}\left(\frac{n}{2}\right)=1,2, \ldots$ comes from $2 p \times 2 p C$ matrix. For the charged component, with $T_{3}=m$, where, $m=n / 2, n / 2-1, \ldots,-n / 2$, we have,

$$
m_{(m)}^{2}=M_{0}^{2}+\frac{1}{2}\left(\alpha-\frac{1}{2} \beta m\right) v^{2} .
$$

Moreover, because of the $\gamma$ term, there will be mixing between components carrying same amount of charge. A component of the multiplet is denoted as $\left|J, T_{3}\right\rangle$. Components with $\left|\frac{n}{2}, m\right\rangle$ and $\left|\frac{n}{2},-(m+1)\right\rangle$ (such that $-m-1 \geq-\frac{n}{2}$ ) will have positive and negative 
charge $Q=m+\frac{1}{2}$ respectively. Now $\langle\Phi\rangle^{T} \epsilon \tau^{a}\langle\Phi\rangle$ gives $\frac{v^{2}}{2 \sqrt{2}}$. Therefore, the mixing matrix between components with charge $|Q|$ is,

$$
M_{Q}^{2}=\left(\begin{array}{cc}
m_{(m)}^{2} & \frac{\gamma v^{2}}{4} \sqrt{\left(\frac{n}{2}-m\right)\left(\frac{n}{2}+m+1\right)} \\
\frac{\gamma v^{2}}{4} \sqrt{\left(\frac{n}{2}-m\right)\left(\frac{n}{2}+m+1\right)} & m_{(-m-1)}^{2}
\end{array}\right)
$$

And the mass eigenstates are,

$$
\begin{aligned}
& \Delta_{1}^{\prime Q}=\cos \theta_{Q} \Delta_{(m)}^{Q}+\sin \theta_{Q} \Delta_{(-m-1)}^{* Q} \\
& \Delta_{2}^{\prime Q}=-\sin \theta_{Q} \Delta_{(m)}^{Q}+\cos \theta_{Q} \Delta_{(-m-1)}^{* Q}
\end{aligned}
$$

where we have

$$
\tan 2 \theta_{Q}=\frac{2\left(M_{Q}^{2}\right)_{12}}{\left(M_{Q}^{2}\right)_{11}-\left(M_{Q}^{2}\right)_{22}}
$$

Note that the real fermionic multiplet is degenerate at the tree level. However, there is a small splitting between the charged and neutral component due to radiative correction which is $O(100 \mathrm{MeV})$ [46]. This splitting is needed in order to treat the neutral fermion as the dark matter candidate.

\subsection{Neutrino mass generation}

The light neutrino masses are generated at one-loop level as shown in figure 1. The neutrino mass matrix is expressed as

$$
\begin{aligned}
& \left(m_{\nu}\right)_{\alpha \beta}=\sum_{i=1}^{3} \frac{y_{\alpha i} y_{i \beta} M_{F_{i}}}{16 \pi^{2}}\left\{C_{\frac{1}{2}, 0,-\frac{1}{2}}^{2}\left[\frac{m_{S}^{2}}{m_{S}^{2}-m_{F_{i}}^{2}} \ln \frac{m_{S}^{2}}{m_{F_{i}}^{2}}-\frac{m_{A}^{2}}{m_{A}^{2}-m_{F_{i}}^{2}} \ln \frac{m_{A}^{2}}{m_{F_{i}}^{2}}\right]\right. \\
& \left.+\sum_{Q \neq 0} C_{\frac{1}{2}, m+\frac{1}{2}, m} C_{\frac{1}{2},-m-\frac{1}{2},-m-1} R_{1, m} R_{2,-m-1}\left[\frac{m_{Q, 1}^{2}}{m_{Q, 1}^{2}-m_{F_{i}}^{2}} \ln \frac{m_{Q, 1}^{2}}{m_{F_{i}}^{2}}-\frac{m_{Q, 2}^{2}}{m_{Q, 2}^{2}-m_{F_{i}}^{2}} \ln \frac{m_{Q, 2}^{2}}{m_{F_{i}}^{2}}\right]\right\} \\
& \quad=\left(y^{T} \Lambda y\right)_{\alpha \beta}
\end{aligned}
$$

Here $C_{m_{1}, m_{2}, m_{3}}$ is the Clebsh-Gordon (CG) coefficient and $m_{1}, m_{2}$ and $m_{3}$ are the $T_{3}$ eigenvalues of lepton doublet, fermion and scalar multiplet respectively. Non-zero CG coefficient requires $m_{1}+m_{3}=m_{2}$. Also $R_{i, m}$ is the element of the rotation matrix that mixes the two scalar components with same charge $|Q|$ and $m_{Q, i}^{2}$ are the corresponding mass eigenvalues. Moreover, $\Lambda_{i}$ is the loop function,

$$
\begin{aligned}
\Lambda_{i}= & \frac{M_{F_{i}}}{16 \pi^{2}}\left\{C_{\frac{1}{2}, 0,-\frac{1}{2}}^{2}\left[\frac{m_{S}^{2}}{m_{S}^{2}-m_{F_{i}}^{2}} \ln \frac{m_{S}^{2}}{m_{F_{i}}^{2}}-\frac{m_{A}^{2}}{m_{A}^{2}-m_{F_{i}}^{2}} \ln \frac{m_{A}^{2}}{m_{F_{i}}^{2}}\right]+\sum_{Q \neq 0} C_{\frac{1}{2}, m+\frac{1}{2}, m} C_{\frac{1}{2},-m-\frac{1}{2},-m-1}\right. \\
& \left.R_{1, m} R_{2,-m-1}\left[\frac{m_{Q, 1}^{2}}{m_{Q, 1}^{2}-m_{F_{i}}^{2}} \ln \frac{m_{Q, 1}^{2}}{m_{F_{i}}^{2}}-\frac{m_{Q, 2}^{2}}{m_{Q, 2}^{2}-m_{F_{i}}^{2}} \ln \frac{m_{Q, 2}^{2}}{m_{F_{i}}^{2}}\right]\right\}
\end{aligned}
$$



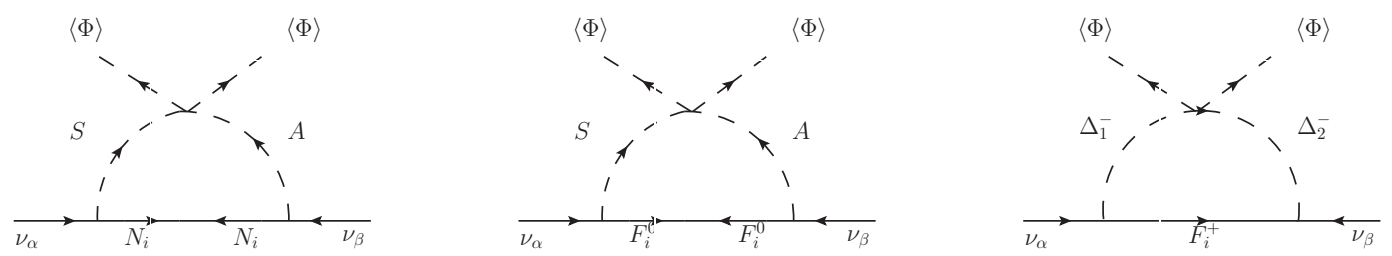

Figure 1. Neutrino mass generation in the inert doublet (first figure from the left) and the quartet (second and third figures).

Therefore the neutrino mass at one loop in the doublet case is given by

$$
\left(m_{\nu}\right)_{\alpha \beta}^{\text {doublet }}=\sum_{i=1}^{3} \frac{y_{\alpha i} y_{i \beta} M_{N_{i}}}{16 \pi^{2}}\left[\frac{m_{S}^{2}}{m_{S}^{2}-m_{N_{i}}^{2}} \ln \frac{m_{S}^{2}}{m_{N_{i}}^{2}}-\frac{m_{A}^{2}}{m_{A}^{2}-m_{N_{i}}^{2}} \ln \frac{m_{A}^{2}}{m_{N_{i}}^{2}}\right]
$$

where $M_{N_{i}}$ is the mass of the i-th right handed neutrino. When $m_{S}^{2} \sim m_{A}^{2} \equiv m_{0}^{2}$ then eq. (2.14) gets simplified

$$
\left(m_{\nu}\right)_{\alpha \beta}^{\text {doublet }}=\sum_{i=1}^{3} \frac{y_{\alpha i} y_{i \beta} \gamma v^{2}}{16 \pi^{2} M_{N_{i}}}\left[\frac{m_{N_{i}}^{2}}{m_{0}^{2}-m_{N_{i}}^{2}}+\left(\frac{m_{N_{i}}^{2}}{m_{0}^{2}-m_{N_{i}}^{2}}\right)^{2} \ln \frac{m_{N_{i}}^{2}}{m_{0}^{2}}\right]
$$

On the other hand, the neutrino mass matrix in the quartet case is given by

$$
\left(M_{\nu}\right)_{\alpha \beta}^{\text {quartet }}=\sum_{i=1}^{2} y_{\alpha i} \Lambda_{i} y_{i \beta}
$$

with the loop factor,

$$
\begin{aligned}
\Lambda_{i}^{\text {quartet }}= & \frac{1}{3(4 \pi)^{2}} M_{F i}\left[\frac{m_{S}^{2}}{m_{S}^{2}-M_{F i}^{2}} \ln \frac{m_{S}^{2}}{M_{F i}^{2}}-\frac{m_{A}^{2}}{m_{A}^{2}-M_{F i}^{2}} \ln \frac{m_{A}^{2}}{M_{F i}^{2}}\right] \\
& +\frac{1}{6(4 \pi)^{2}} \sin 2 \theta M_{F i}\left[\frac{m_{\Delta_{1}^{+}}^{2}}{m_{\Delta_{1}^{+}}^{2}-M_{F i}^{2}} \ln \frac{m_{\Delta_{1}^{+}}^{2}}{M_{F i}^{2}}-\frac{m_{\Delta_{2}^{+}}^{2}}{m_{\Delta_{2}^{+}}^{2}-M_{F i}^{2}} \ln \frac{m_{\Delta_{2}^{+}}^{2}}{M_{F i}^{2}}\right]
\end{aligned}
$$

Explicit expressions of masses in the inert doublet and quartet models are included in appendix A.

The neutrino mass matrix can be diagonalized as

$$
U_{\mathrm{PMNS}}^{T} m_{\nu} U_{\mathrm{PMNS}} \equiv \hat{m}_{\nu}
$$

where

$$
U_{\mathrm{PMNS}}=\left(\begin{array}{ccc}
c_{12} c_{13} & s_{12} c_{13} & s_{13} e^{i \delta} \\
-s_{12} c_{23}-c_{12} s_{23} s_{13} e^{-i \delta} & c_{12} c_{23}-s_{12} s_{23} s_{13} e^{-i \delta} & s_{23} c_{13} \\
s_{12} s_{23}-c_{12} c_{23} s_{13} e^{-i \delta} & -c_{12} s_{23}-s_{12} c_{23} s_{13} e^{-i \delta} & c_{23} c_{13}
\end{array}\right) \times\left(\begin{array}{ccc}
1 & 0 & 0 \\
0 & e^{i \alpha / 2} & 0 \\
0 & 0 & e^{i \beta / 2}
\end{array}\right)
$$

Here, $c_{i j}=\cos \theta_{i j}, s_{i j}=\sin \theta_{i j}, \delta$ is the Dirac phase and $\alpha, \beta$ are the Majorana phases. 
The Yukawa matrix $y_{i \alpha}(\alpha=e, \mu, \tau)$ is expressed using the Casas-Ibarra parametrization [49] so that the chosen parameter space automatically satisfies the low energy neutrino parameters,

$$
y=\sqrt{\Lambda}^{-1} R \sqrt{\hat{m}_{\nu}} U_{\mathrm{PMNS}}^{\dagger}
$$

where $R$ is a complex orthogonal matrix.

\subsection{Perturbativity}

If there are $\mathrm{N}$ generations of right handed fermion multiplet, perturbativity of the Yukawa gives the following constraint $[50,51]$

$$
\operatorname{Tr}\left(y^{\dagger} y\right)=\sum_{i=1}^{3} \sum_{j=1}^{N}\left|R_{i j}\right|^{2} \frac{\hat{m}_{\nu_{i}}}{\Lambda_{j}} \lesssim O(1)
$$

If $R$ is taken to be real, the constraint translates into the largest ratio, $\frac{\hat{m}_{\nu_{i}}}{\Lambda_{j}} \lesssim O(1)$, whereas for the general case when $R$ is complex, each entry will be bounded as $\left|R_{i j}\right| \lesssim$ $\sqrt{\frac{\Lambda_{j}}{3 N \hat{m}_{\nu_{i}}}}$.

\section{Lepton flavor violating processes}

In this section we have presented the relevant analytical formulas of LFV processes for the doublet and quartet case. In the standard model due to the GIM suppression the rate of $\mu \rightarrow e \gamma$ becomes $\sim 10^{-54}$ thus negligible. On the other hand the presence of heavy right handed neutrino that mixes with left handed (LH) neutrinos, spoils the GIM suppression and one could obtain the rate which can be probed by experiment [52-58]. In inert scalar models, $Z_{2}$ symmetry forbids the mixing between $\mathrm{LH}$ and $\mathrm{RH}$ neutrinos but the enhancements in the LFV processes are provided by the $C^{ \pm}-N_{R_{i}}$ loops in the doublet and $\Delta-F_{i}$ loops in the quartet model. We have focused on three LFV processes: $\mu \rightarrow e \gamma$, $\mu \rightarrow e e \bar{e}$ and $\mu-e$ conversion in nuclei in this paper as they have the most stringent limits from the experiments.

\section{$3.1 \mu \rightarrow e \gamma$}

The branching ratio for $\mu \rightarrow e \gamma$, normalized by $\operatorname{Br}\left(\mu \rightarrow e \overline{\nu_{e}} \nu_{\mu}\right)$, is $[27,59]$

$$
\operatorname{Br}(\mu \rightarrow e \gamma)=\frac{3(4 \pi)^{3} \alpha_{e m}}{4 G_{F}^{2}}\left|A_{D}\right|^{2} \operatorname{Br}\left(\mu \rightarrow e \nu_{\mu} \overline{\nu_{e}}\right)
$$

where $A_{D}$ is the dipole form factor. The Feynman diagrams of one-loop contributions by the doublet and quartet to the $\mu e \gamma$ vertex that enters into the dipole form factor calculation, are given in figure 9 .

The contributions from the doublet is the following,

$$
A_{D}^{\text {doublet }}=\sum_{i=1}^{3} \frac{y_{e i}^{*} y_{i \mu}}{32 \pi^{2}} \frac{1}{m_{C}^{2}} F^{(n)}\left(x_{i \sigma}\right)
$$


Here $F^{(n)}(x)$ is the loop function given in the appendix B and $x_{i \sigma}=m_{N_{i}}^{2} / m_{\sigma}^{2}$, where $\sigma=C^{+}$. On the other hand, the quartet contribution will have two parts

$$
A_{D}^{\text {quartet }}=A_{D(n)}^{\text {quartet }}+A_{D(c)}^{\text {quartet }}
$$

where $A_{D(n)}^{\text {quartet }}$ is the contribution of the neutral component and $A_{D(c)}^{\text {quartet }}$ is that of the charged component of the fermion triplet. Also, for the notational convenience, we introduce generalized Yukawa coupling $y_{i \alpha \sigma}=y_{i \alpha} C_{\sigma}$ where $C_{\sigma}$ is the corresponding Clebsh Gordon coefficient associated with $\sigma$-th component of the quartet. The two contributions are

$$
A_{D(n)}^{\text {quartet }}=\sum_{i=1}^{3} \sum_{\sigma} \frac{y_{e i \sigma}^{*} y_{i \mu \sigma}}{32 \pi^{2}} \frac{1}{m_{\sigma}^{2}} F^{(n)}\left(x_{i \sigma}\right)
$$

where $x_{i \sigma}=m_{F_{i}^{0}}^{2} / m_{\sigma}^{2}, \sigma=\Delta_{1}^{+}, \Delta_{2}^{+}$. And

$$
A_{D(c)}^{\text {quartet }}=-\sum_{i=1}^{3} \sum_{\sigma} \frac{y_{e i \sigma}^{*} y_{i \mu \sigma}}{32 \pi^{2}} \frac{1}{m_{\sigma}^{2}} F^{(c)}\left(x_{i \sigma}\right)
$$

where $x_{i \sigma}=m_{F_{i}^{ \pm}}^{2} / m_{\sigma}^{2}$, and $\sigma=\Delta^{++}, S, A$.

\section{$3.2 \mu \rightarrow e e \bar{e}$}

Now we turn to $\mu \rightarrow e e \bar{e}$ decay. The branching ratio is given as $[27,59,60]$

$$
\begin{aligned}
\operatorname{Br}(\mu \rightarrow e e \bar{e})= & \frac{3(4 \pi)^{2} \alpha_{e m}^{2}}{8 G_{F}^{2}}\left[\left|A_{N D}\right|^{2}+\left|A_{D}\right|^{2}\left(\frac{16}{3} \ln \frac{m_{\mu}}{m_{e}}-\frac{22}{3}\right)+\frac{1}{6}|B|^{2}\right. \\
& \left.+\frac{1}{3}\left(2\left|F_{Z}^{L}\right|^{2}+\left.F_{Z}^{R}\right|^{2}\right)+\left(-2 A_{N D} A_{D}^{*}+\frac{1}{3} A_{N D} B^{*}-\frac{2}{3} A_{D} B^{*}+\text { h.c }\right)\right] \\
& \times \operatorname{Br}\left(\mu \rightarrow e \overline{\nu_{e}} \nu_{\mu}\right)
\end{aligned}
$$

where $A_{D}$ and $A_{N D}$ are the dipole and non-dipole contribution from the photonic penguin diagrams respectively. Also $B$ represents the contribution from the box diagrams. Moreover, $F_{Z}^{L}$ and $F_{Z}^{R}$ are given as

$$
F_{Z}^{L}=\frac{F_{Z} g_{L}^{l}}{g^{2} m_{Z}^{2} \sin ^{2} \theta_{W}}, \quad F_{Z}^{R}=\frac{F_{Z} g_{R}^{l}}{g^{2} m_{Z}^{2} \sin ^{2} \theta_{W}}
$$

Here, $F_{Z}$ is the Z-penguin contribution and $g_{L}^{l}$ and $g_{R}^{l}$ are the Z-boson coupling to the LH and RH charged leptons respectively. In this model, Higgs penguin contribution will be suppressed by the small electron Yukawa coupling, and therefore we have only considered the photon penguin, Z-boson penguin and box diagrams.

\subsection{1 $\gamma$-penguin contribution}

First let us consider contributions from the photon penguin diagrams. In this case the $\gamma$ line of $\mu e \gamma$ vertex given in figure 9 will have $\bar{e} e$ attached to it. The photonic non-dipole contribution, $A_{N D}$ for the doublet is in the following

$$
A_{N D}^{\text {doublet }}=\sum_{i=1}^{3} \frac{y_{e i}^{*} y_{i \mu}}{96 \pi^{2}} \frac{1}{m_{C}^{2}} G^{(n)}\left(x_{i \sigma}\right)
$$


The photonic non-dipole contribution, for the case of the quartet, will again have two parts,

$$
A_{N D}^{\text {quartet }}=A_{N D(n)}^{\text {quartet }}+A_{N D(c)}^{\text {quartet }}
$$

Here $A_{N D(n)}^{\text {quartet }}$ is the contribution of the neutral component and $A_{N D(c)}^{\text {quartet }}$ is the contribution of the charged component of the fermion triplet.

$$
A_{N D(n)}^{\text {quartet }}=\sum_{i=1}^{3} \sum_{\sigma=\Delta_{1}^{+}, \Delta_{2}^{+}} \frac{y_{e i \sigma}^{*} y_{i \mu \sigma}}{96 \pi^{2}} \frac{1}{m_{\sigma}^{2}} G^{(n)}\left(x_{i \sigma}\right)
$$

where again $x_{i \sigma}=m_{F_{i}^{0}}^{2} / m_{\sigma}^{2}$. And the charged component of fermion triplet contributes as follows,

$$
A_{N D(c)}^{\text {quartet }}=-\sum_{i=1}^{3} \sum_{\sigma} \frac{y_{e i \sigma}^{*} y_{i \mu \sigma}}{96 \pi^{2}} \frac{1}{m_{\sigma}^{2}} G^{(c)}\left(x_{i \sigma}\right)
$$

with $x_{i \sigma}=m_{F_{i}^{ \pm}}^{2} / m_{\sigma}^{2}$, and $\sigma=\Delta^{++}, S, A$. The loop functions $F^{(n)}(x), F^{(c)}(x), G^{(n)}(x)$ and $G^{(c)}(x)$ are given in the appendix B.

\subsubsection{Z-penguin contribution}

Now we focus on the Z-penguin diagram. The Feynman diagrams of one-loop contributions from the doublet and the quartet to the $\mu e Z$ vertex are presented in figure 10 . In Z-penguin diagram, the $Z$ line of $\mu e Z$ vertex will have $\bar{e} e$ line attached to it. For the doublet, the contribution is given by the neutral fermion. Following the formulas given in $[60,62,63]^{1}$

$$
F_{Z(n)}^{\text {doublet }}=-\frac{1}{16 \pi^{2}} \sum_{i=1}^{3} y_{e i}^{*} y_{i \mu}\left[2 g_{Z C^{+} C^{-}} C_{24}\left(m_{N_{i}}, m_{C}, m_{C}\right)+g_{L}^{l} B_{1}\left(m_{N_{i}}, m_{C}\right)\right]
$$

Here, $g_{Z C^{+} C^{-}}$is the $\mathrm{Z}$ boson coupling to $C^{ \pm}$of the doublet and $g_{L}^{l}$ is the $\mathrm{Z}$ boson coupling to LH charged leptons given by

$$
g_{L}^{l}=\frac{g}{\cos \theta_{W}}\left(-\frac{1}{2}+\sin ^{2} \theta_{W}\right)
$$

On the other hand, the quartet contribution is

$$
F_{Z}^{\text {quartet }}=F_{Z(n)}^{\text {quartet }}+F_{Z(c)}^{\text {quartet }}
$$

where the neutral fermion of the triplet contributes as

$$
\begin{aligned}
F_{Z(n)}^{\text {quartet }}= & -\frac{1}{16 \pi^{2}} \sum_{i=1}^{3} \sum_{\sigma_{1}, \sigma_{2}}\left[2 y_{e i \sigma_{1}}^{*} y_{i \mu \sigma_{2}} g_{Z \sigma_{1} \sigma_{2}} C_{24}\left(m_{F_{i}^{0}}, m_{\sigma_{1}}, m_{\sigma_{2}}\right)\right. \\
& \left.+y_{e i \sigma_{1}}^{*} y_{i \mu \sigma_{1}} g_{L}^{l} B_{1}\left(m_{F_{i}^{0}}, m_{\sigma_{1}}\right)\right]
\end{aligned}
$$

\footnotetext{
${ }^{1}$ Ref. [60] contained a mistake in the calculation of Z-penguin diagram which was pointed out in [61]. Subsequently, correct results were presented in [62] and [63]. Moreover, $C_{00}$ of [62] and $C_{24}$ of [63] only differ by an overall minus sign.
} 
where $\sigma_{1,2} \in\left\{\Delta_{1}^{+}, \Delta_{2}^{+}\right\}$and $g_{Z \sigma_{1} \sigma_{2}}$ is the $\mathrm{Z}$ boson coupling to $\sigma_{1}$ and $\sigma_{2}$ scalars of the quartet. The charged fermion of the triplet has the following contribution

$$
\begin{aligned}
F_{Z(c)}^{\text {quartet }}= & -\frac{1}{16 \pi^{2}} \sum_{i=1}^{3} \sum_{\sigma_{1}, \sigma_{2}}\left\{y _ { \text { ei } \sigma _ { 1 } } ^ { * } y _ { i \mu \sigma _ { 1 } } g _ { Z F _ { i } ^ { \pm } } \overline { F _ { i } ^ { \pm } } \left[\left(2 C_{24}\left(m_{\sigma_{1}}, m_{F_{i}^{ \pm}}, m_{F_{i}^{ \pm}}\right)+\frac{1}{2}\right)\right.\right. \\
& \left.+m_{F_{i}^{ \pm}}^{2} C_{0}\left(m_{\sigma_{1}}, m_{F_{i}^{ \pm}}, m_{F_{i}^{ \pm}}\right)\right]+2 y_{\text {ei } \sigma_{1}}^{*} y_{i \mu \sigma_{2}} g_{Z \sigma_{1} \sigma_{2}} C_{24}\left(m_{F_{i}^{ \pm}}, m_{\sigma_{1}}, m_{\sigma_{2}}\right) \\
& \left.+y_{\text {ei } \sigma_{1}}^{*} y_{i \mu \sigma_{1}} g_{L}^{l} B_{1}\left(m_{F_{i}^{ \pm}}, m_{\sigma_{1}}\right)\right\}
\end{aligned}
$$

Here $\sigma_{1}$ and $\sigma_{2}$ range over the $S, A, \Delta^{++}$, and $g_{Z F_{i}^{ \pm}} \overline{F_{i}^{ \pm}}$is the coupling of $\mathrm{Z}$ boson to charged fermions. Moreover, $B_{1}, C_{0}$ and $C_{24}$ are the loop functions, adopted from [60, 62, 63], presented in the appendix B. As $B_{1}$ and $C_{24}$ arise from divergent loop integrals, for large $M$,

$$
C_{24}(M, m, m) \rightarrow \frac{1}{4} \ln \frac{M^{2}}{\mu^{2}}, B_{1} \rightarrow \frac{1}{2} \ln \frac{M^{2}}{\mu^{2}}
$$

Therefore the combination $2 x C_{24}+y B_{1}$ in Z-penguin contribution eq. (3.15) or in eq. (3.16) is vanishing at very large mass $M$ when there are specific relations set by group theoretical requirements in vertex factors $x$ and $y$.

\subsubsection{Box contribution}

Lastly the box contribution for the doublet case, presented in figure 11, is [60]

$$
e^{2} B_{(n)}^{\text {doublet }}=\frac{1}{16 \pi^{2}} \sum_{i, j=1}^{3}\left[\frac{\tilde{D}_{0}}{2} y_{e i}^{*} y_{i \mu} y_{e j}^{*} y_{j e}+D_{0} m_{N_{i}} m_{N_{j}} y_{e i}^{*} y_{e i}^{*} y_{j \mu} y_{j e}\right]
$$

where, $\tilde{D}_{0}=\tilde{D}_{0}\left(m_{N_{i}}, m_{N_{j}}, m_{C}, m_{C}\right)$ and $D_{0}=D_{0}\left(m_{N_{i}}, m_{N_{j}}, m_{C}, m_{C}\right)$ are loop functions given in the appendix $\mathrm{B}$.

For the quartet case, the contribution of the box diagram can be written as

$$
B^{\text {quartet }}=B_{(n)}^{\text {quartet }}+B_{(c)}^{\text {quartet }}
$$

with $B_{(n)}^{\text {quartet }}$ is the contribution due to the neutral fermions and it is given by

$$
e^{2} B_{(n)}^{\text {quartet }}=\frac{1}{16 \pi^{2}} \sum_{i, j=1}^{3} \sum_{\sigma_{1}, \sigma_{2}}\left[\frac{\tilde{D}_{0}}{2} y_{e i \sigma_{1}}^{*} y_{i \mu \sigma_{2}} y_{e j \sigma_{2}}^{*} y_{j e \sigma_{1}}+D_{0} m_{F_{i}^{0}} m_{F_{j}^{0}} y_{e i \sigma_{1}}^{*} y_{e i \sigma_{2}}^{*} y_{j \mu \sigma_{2}} y_{j e \sigma_{1}}\right]
$$

where, $\tilde{D}_{0}=\tilde{D}_{0}\left(m_{F_{i}^{0}}, m_{F_{j}^{0}}, m_{\sigma_{1}}, m_{\sigma_{2}}\right)$ and $D_{0}=D_{0}\left(m_{F_{i}^{0}}, m_{F_{j}^{0}}, m_{\sigma_{1}}, m_{\sigma_{2}}\right)$. Here, $\sigma_{1,2}$ ranges over $\Delta_{1}^{+}$and $\Delta_{2}^{+}$.

The term $B_{(c)}^{\text {quartet }}$ corresponds to the contribution of the charged fermions and it reads

$$
e^{2} B_{(c)}^{\text {quartet }}=\frac{1}{16 \pi^{2}} \sum_{i, j=1}^{3} \sum_{\sigma_{1}, \sigma_{2}} \frac{\tilde{D}_{0}}{2} y_{e i \sigma_{1}}^{*} y_{i \mu \sigma_{2}} y_{e j \sigma_{2}}^{*} y_{j e \sigma_{1}}
$$

Here, $\tilde{D}_{0}=\tilde{D}_{0}\left(m_{F_{i}^{ \pm}}, m_{F_{j}^{ \pm}}, m_{\sigma_{1}}, m_{\sigma_{2}}\right)$ and $\sigma_{1,2}$ ranges over $\Delta^{++}, S, A$. 


\section{$3.3 \mu-e$ conversion in nuclei}

The conversion rate, normalized by the muon capture rate is $[27,64-66]$

$$
\begin{aligned}
\mathrm{CR}(\mu-e, \text { Nucleus })= & \frac{p_{e} E_{e} m_{\mu}^{3} G_{F}^{2} \alpha_{e m}^{3} Z_{\text {eff }}^{4} F_{p}^{2}}{8 \pi^{2} Z \Gamma_{\text {capt }}}\left\{\left|(Z+N)\left(g_{L V}^{(0)}+g_{L S}^{(0)}\right)+(Z-N)\left(g_{L V}^{(1)}+g_{L S}^{(1)}\right)\right|^{2}\right. \\
& \left.+\left|(Z+N)\left(g_{R V}^{(0)}+g_{R S}^{(0)}\right)+(Z-N)\left(g_{R V}^{(1)}+g_{R S}^{(1)}\right)\right|^{2}\right\}
\end{aligned}
$$

Here, $Z$ and $N$ are the number of protons and neutrons in the nucleus, $Z_{\text {eff }}$ is the effective atomic charge, $F_{p}$ is the nuclear matrix element and $\Gamma_{\text {capt }}$ represents the total muon capture rate. $p_{e}$ and $E_{e}$ are the momentum and energy of the electron (taken as $\sim m_{\mu}$ in the numerical evaluation). $g_{X K}^{(0)}$ and $g_{X K}^{(1)}(X=L, R$ and $K=V, S)$ in the above expression are given as

$$
\begin{aligned}
& g_{X K}^{(0)}=\frac{1}{2} \sum_{q=u, d, s}\left(g_{X K(q)} G_{K}^{(q, p)}+g_{X K(q)} G_{K}^{(q, n)}\right) \\
& g_{X K}^{(1)}=\frac{1}{2} \sum_{q=u, d, s}\left(g_{X K(q)} G_{K}^{(q, p)}-g_{X K(q)} G_{K}^{(q, n)}\right)
\end{aligned}
$$

$g_{X K(q)}$ are the couplings in the effective Lagrangian describing $\mu-e$ conversion,

$$
\mathcal{L}_{\text {eff }}=-\frac{G_{F}}{\sqrt{2}} \sum_{q}\left\{\left[g_{L S(q)} \bar{e}_{L} \mu_{R}+g_{R S(q)} \bar{e}_{R} \mu_{L}\right] \bar{q} q+\left[g_{L V(q)} \bar{e}_{L} \gamma^{\mu} \mu_{L}+g_{R V(q)} \bar{e}_{R} \gamma^{\mu} \mu_{R}\right] \bar{q} \gamma_{\mu} q\right\}
$$

$G^{(q, p)}, G^{(q, n)}$ are the numerical factors that arise when quark matrix elements are replaced by the nucleon matrix elements,

$$
\left\langle p\left|\bar{q} \Gamma_{K} q\right| p\right\rangle=G_{K}^{(q, p)} \bar{p} \Gamma_{K} p, \quad\left\langle n\left|\bar{q} \Gamma_{K} q\right| n\right\rangle=G_{K}^{(q, n)} \bar{n} \Gamma_{K} n
$$

For the inert scalar model, the $\mu-e$ conversion rate receives the $\gamma, \mathrm{Z}$ and Higgs penguin contributions. In $\gamma$ and $\mathrm{Z}$ penguin diagrams, $\bar{q} q(\mathrm{q}=\mathrm{u}, \mathrm{d}, \mathrm{s})$ line is attached to $\gamma$ line of $\mu e \gamma$ vertex and $\mathrm{Z}$ boson line of $\mu e Z$ vertex respectively. It doesn't receive any box contribution because there is no coupling between inert scalars and quarks because of the $Z_{2}$ symmetry. Moreover, Higgs penguin contribution is small compared to $\gamma$ and $\mathrm{Z}$ penguin diagrams because of small Yukawa couplings thus neglected in our numerical analysis. The relevant effective coupling for the conversion in the inert scalar model is

$$
\begin{aligned}
g_{L V(q)} & =g_{L V(q)}^{\gamma}+g_{L V(q)}^{Z} \\
g_{R V(q)} & =\left.g_{L V(q)}\right|_{L \leftrightarrow R} \\
g_{L S(q)} & \approx 0, \quad g_{R S(q)} \approx 0
\end{aligned}
$$

The relevant couplings are

$$
\begin{aligned}
& g_{L V(q)}^{\gamma}=\frac{\sqrt{2}}{G_{F}} e^{2} Q_{q}\left(A_{N D}-A_{D}\right) \\
& g_{L V(q)}^{Z}=-\frac{\sqrt{2}}{G_{F}} \frac{g_{L}^{q}+g_{R}^{q}}{2} \frac{F_{Z}}{m_{Z}^{2}}
\end{aligned}
$$


Here $Q_{q}$ is the electric charge of the quarks and $\mathrm{Z}$ boson couplings to the quarks are

$$
g_{L}^{q}=\frac{g}{\cos \theta_{W}}\left(T_{3}^{q}-Q_{q} \sin ^{2} \theta_{W}\right), \quad g_{R}^{q}=-\frac{g}{\cos \theta_{W}} Q_{q} \sin ^{2} \theta_{W}
$$

Also the relevant numerical factors for nucleon matrix elements are

$$
G_{V}^{(u, p)}=G_{V}^{(d, n)}=2, \quad G_{V}^{(d, p)}=G_{V}^{(u, n)}=1
$$

\section{Results and discussion}

In this section we have presented our numerical results and discussed the phenomenological implications of those results for larger scalar multiplets. But before presenting the results, we have listed all the constraints regarding dark matter and collider searches so that our analysis can focus on parameter space for where both inert doublet and quartet models are viable.

There are two possible dark matter (DM) candidates in the inert scalar models. In the doublet model they are the lightest right handed neutrino, $N_{1}$ and the lightest neutral scalar, $S$ of the doublet. On the other hand, in the quartet model the neutral component of the lightest fermion triplet, $F_{1}^{0}$ and the lightest neutral scalar, $S$ of the quartet can play the dark matter role. In both cases fermionic and scalar DM give rise to different phenomenology. In this preliminary study of comparing different LFV rates in inert scalar models, we have chosen the scalar as the DM particle and used the constraints associated with it in our analysis.

\subsection{Constraints and parameter space}

\subsubsection{Collider constraints}

For the doublet scalar, the collider searches have put the following mass constraints, $m_{C^{+}} \gtrsim$ $100 \mathrm{GeV}, m_{S} \gtrsim 65-80 \mathrm{GeV}$ and $m_{A} \gtrsim 140 \mathrm{GeV}$ [17-21]. Although there hasn't been any collider studies on the quartet, one can recast the constraints of the doublet case onto the quartet. As the quartet scalar has the cascade decay channel, we can expect multilepton final states along with missing transverse energy similar to doublet. Therefore, the mass constraints for quartet, compatible with bounds on electroweak precision observable [67], are $m_{\Delta_{1,2}}, m_{\Delta^{++}} \gtrsim 100 \mathrm{GeV}, m_{S} \gtrsim 65-80 \mathrm{GeV}$ and $m_{A} \gtrsim 140 \mathrm{GeV}$. Considering $S$ as the DM also set the mass hierarchy in quartet components: $m_{S}<m_{\Delta_{1}^{+}}<m_{\Delta^{++}}<m_{\Delta_{2}^{+}}<m_{A}$. In contrast, the scalar masses in the $\mathrm{TeV}$ scale for both doublet and quartet are fairly unconstrained.

In the case of fermions, the masses of $\mathrm{RH}$ neutrino in the doublet case are not constrained by current collider data. In contrast, fermion triplet of the quartet case, having gauge interaction, will have an accessible collider signature. In [68] the mass of the charged component of the triplet is excluded up to $270 \mathrm{GeV}$ with $8 \mathrm{TeV} 20.3 \mathrm{fb}^{-1} \mathrm{LHC}$ data. More-

over, in [69] it was shown that the projected reach for $14 \mathrm{TeV}$ collider with $3 \mathrm{ab}^{-1}$ luminosity (High luminosity LHC phase) would be $M_{F} \lesssim 500 \mathrm{GeV}$, for (future) $100 \mathrm{TeV}$ pp collider with $3 \mathrm{ab}^{-1}$ luminosity in mono-jet searches, $M_{F} \lesssim 1.3 \mathrm{TeV}$, and with $30 \mathrm{ab}^{-1}$ luminosity, $M_{F} \lesssim 1.7 \mathrm{TeV}$. 


\subsubsection{DM constraints}

The dark matter density of the universe measured by Planck collaboration is $\Omega_{D M} h^{2}=$ $0.1196 \pm 0.0031(68 \% \mathrm{CL})[70]$. In the inert scalar model, there are two viable mass region of scalar DM. They are the low mass region $\left(m_{S}<m_{W}\right)$ and the high mass region $\left(m_{S} \gg\right.$ $\left.m_{W}\right)$. The low mass DM region of doublet model has been extensively studied. In addition, same region for DM in the quartet was addressed in [22] where it was shown that it is harder to achieve low mass dark matter with correct relic density compared to the doublet because, for most of the parameter space, bounds on electroweak $T$ parameter sets the mass of single charged component, $\Delta_{1}^{+}$close to the DM mass and therefore it is not only in tension with collider bounds but also opens up coannihilation channel and leads to a sub-dominant DM in the universe.

In the high mass region of the doublet, as shown in [71], the DM mass starts from a lower bound of $m_{0}=534 \pm 25 \mathrm{GeV}$ (where the thermal freeze-out only happens through the gauge interaction) to $20 \mathrm{TeV}$ if the higgs-scalar coupling, $\lambda_{S} \lesssim 2 \pi$. The maximal mass splitting compatible with correct relic density, are

$$
\left|m_{A}-m_{S}\right| \lesssim 16.9 \mathrm{GeV},\left|m_{C^{+}}-m_{S}\right| \lesssim 14.6 \mathrm{GeV}
$$

when $m_{S} \sim O(5 \mathrm{TeV})$.

In the case of high mass region for the quartet, we have used FeynRules [72] to generate the model files for MicrOMEGAS [73] and have found out that the DM mass starts from a lower bound of $2.46 \mathrm{TeV}$ (freeze out only through gauge interaction) ${ }^{2}$ to upper bound of $14 \mathrm{TeV}$ set again by $\lambda_{S} \lesssim 2 \pi$ bound. In this case, the mass splitting between the DM and other components are

$$
\begin{aligned}
\left|m_{A}-m_{S}\right| & \lesssim 16 \mathrm{GeV},\left|m_{\Delta_{2}^{+}}-m_{S}\right| \lesssim 14 \mathrm{GeV} \\
\left|m_{\Delta^{++}}-m_{S}\right| & \lesssim 12 \mathrm{GeV},\left|m_{\Delta_{1}^{+}}-m_{S}\right| \lesssim 1 \mathrm{GeV}
\end{aligned}
$$

when $m_{S} \sim O(5 \mathrm{TeV})$. Figure 2 presents the $m_{S}-\lambda_{S}$ plane with allowed region for both doublet and quartet scalar DM by the relic density and direct detection bound [74]. Here, $\lambda_{S}$ is effective coupling of $S$ to Higgs field as can be seen in eq. (2.6). From figure 2, we can see that there is an overlapping region on the plane where doublet and quartet DM satisfy the constraints simultaneously.

The $\gamma$ coupling which controls the mass splitting between scalar (DM) and pseudoscalar component, has the range $\gamma \in\left[10^{-9}, 2.7\right]$ for the doublet and $\gamma \in\left[10^{-9}, 1.36\right]$ to be consistent with the relic density. But it gets another constraint from bounds on DM inelastic scattering with nuclei. If the typical velocity of a DM particle, $\chi$ is $\beta_{\chi} c \sim 220 \mathrm{~km} / \mathrm{sec}$, the inelastic scattering is kinematically forbidden if the splitting $\Delta_{\chi}$ between DM and the next to lightest component is larger,

$$
\Delta_{\chi}>\frac{\beta_{\chi}^{2} m_{\chi} M_{\text {nucleus }}}{2\left(m_{\chi}+M_{\text {nucleus }}\right)}
$$

\footnotetext{
${ }^{2}$ without considering the Sommerfeld enhancement
} 

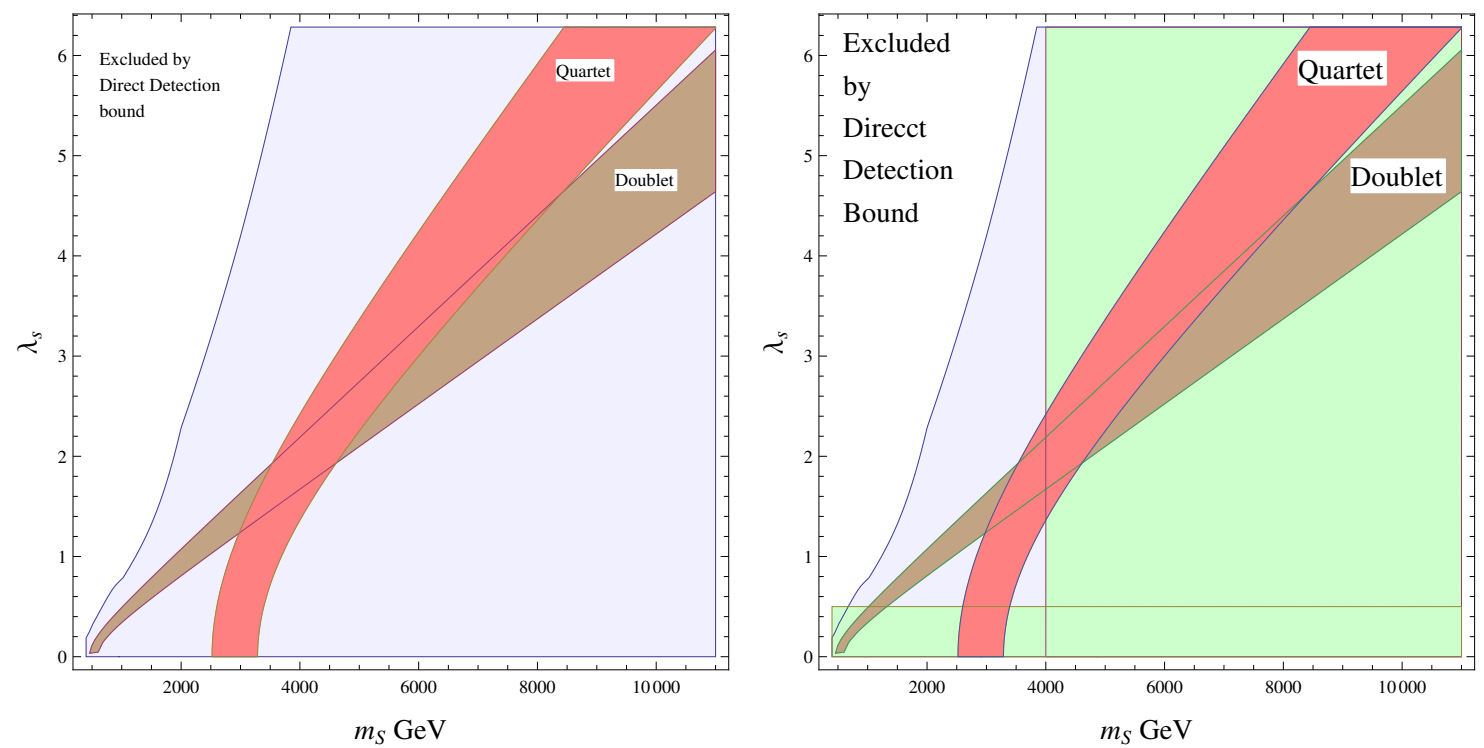

Figure 2. Correlation between the mass of the DM, $m_{S}$ and the effective coupling between the Higgs and the DM, $\lambda_{S}$ for the doublet and quartet case. Here, the white region is excluded by the direct detection bound from the LUX collaboration [74]. The left figure represents the correlation without taking into account the Sommerfeld enhancement in the thermal freeze-out. In the right figure, for the green shaded region, Sommerfeld enhancement is not negligible.

Therefore one would require, $\gamma \gtrsim 10^{-5}$ to kinematically forbid the inelastic scattering of scalar DM with $\mathrm{O}(\mathrm{TeV})$ mass. As the inelastic scattering is mediated by the exchange of $\mathrm{Z}$ boson and the scattering cross section is in the order of $10^{-40}-10^{-39} \mathrm{~cm}^{2}$, which is much larger than the direct detection bounds, the allowed range of $\gamma$ for doublet and quartet DM are $\gamma \in\left[10^{-5}, 2.7\right]$ and $\gamma \in\left[10^{-5}, 1.36\right]$, respectively.

\subsubsection{Gamma ray constraints and Sommerfeld enhancement}

Compared to the collider searches and DM direct detection experiments, indirect detection can set limits on the inert scalar $\mathrm{DM}$ at the $\mathrm{TeV}$ mass range because of a certain enhancement in the annihilation cross sections.

At small relative velocity, two particles interacting via a long range force receive nonperturbative enhancement in the interaction cross section which is known as Sommerfeld enhancement [75]. When the mass of the DM is much larger than the mass of $\mathrm{W}$ and $\mathrm{Z}$ bosons, the electroweak interaction effectively behaves like a long range force, thus pair annihilation cross sections of the DM also receive Sommerfeld enhancements as pointed in [76-78]. At present, as the relative velocity of DM is about $10^{-3}$, Sommerfeld enhancement significantly boosts the indirect detection signals, specially the gamma rays produced from the DM annihilation and put stringent constraint on the DM in the light of the experimental observations. In fact it was shown for the case of wino dark matter [81,82] and minimal DM models (5-plet fermion and 7-plet scalar with zero hypercharge) [83-85] (and references therein) that they are highly constrained to be the dominant DM of the universe by the experimental limits on gamma ray spectrum due to the Sommerfeld enhancement in the pair annihilation cross section. 
Having electroweak charge, the heavy DM component of the inert scalar multiplet is also expected to have enhancements in both weak and scalar interactions. Although the full treatment of Sommerfeld enhancement for inert scalar model is beyond the scope of this work, following $[79,80]$, we introduce the dimensionless parameters to curve out the regions of the parameter space where the enhancement takes place and where the enhancement is negligible. The parameters are, $\epsilon_{v_{\mathrm{DM}}}=\left(v_{\mathrm{DM}} / c\right) / \alpha, \epsilon_{\phi}=\left(m_{\phi} / m_{\mathrm{DM}}\right) / \alpha$ and $\epsilon_{\delta}=\sqrt{2 \delta / m_{\mathrm{DM}}} / \alpha$. Here $v_{\mathrm{DM}}$ is the relative velocity of the DM particle, $m_{\phi}$ is the mass of the gauge boson carrying the force, $\delta$ is the mass splitting between the DM and the next to lightest charged component of the multiplet and $\alpha$ is the coupling constant of the relevant interaction. It was shown in [80] that the Sommerfeld enhancement is relevant if $\epsilon_{v_{\mathrm{DM}}}, \epsilon_{\phi}, \epsilon_{\delta} \lesssim 1$. On the other hand, it is negligible for the region of parameter space where any of $\epsilon_{v_{\mathrm{DM}}}, \epsilon_{\phi}, \epsilon_{\delta}>1$.

In the case of the minimal DM models, the processes contributing to the gamma spectrum from DM annihilation are, DM DM $\rightarrow W^{+} W^{-}, Z Z$ where the decay and fragmentation of $\mathrm{W}$ and $\mathrm{Z}$ pairs produce secondary photons and $\mathrm{DM} \mathrm{DM} \rightarrow \gamma \gamma, \gamma Z$ producing line spectrum of mono energetic photons. The Sommerfeld enhancement takes place when the DM-DM two particle state changes into $\mathrm{DM}^{+} \mathrm{DM}^{-}$two particle state, where $\mathrm{DM}^{ \pm}$is the next to lightest charged state, by exchanging $\mathrm{W}$ boson and subsequently charged states annihilate. For the minimal DM case, the DM and next to lightest charged state is almost degenerate (only loop induced mass splitting of the $O(100) \mathrm{MeV}$ ), so $\epsilon_{\delta}<1$ for $\alpha_{w}=1 / 30$ and $\mathrm{TeV}$ scale DM and one can have Sommerfeld enhanced annihilation cross section. On the other hand, for the inert scalar models, the following terms in the scalar potential

$$
V \supset \beta \Phi^{\dagger} \tau^{a} \Phi \Delta^{\dagger} T^{a} \Delta+\gamma\left[\left(\Phi^{T} \epsilon \tau^{a} \Phi\right)\left(\Delta^{T} C T^{a} \Delta\right)^{\dagger}+h . c\right]
$$

can split the DM component and other charged component of the multiplet after electroweak symmetry breaking. For example, for quartet, when $m_{S}=3 \mathrm{TeV}$ and $\delta=$ $m_{\Delta_{1}^{+}}-m_{S}=1.5 \mathrm{GeV}, \epsilon_{\delta}$ is 1.001 . In addition, from figure 3 , we can see that the bounds on electroweak precision observables allow maximum mass splitting to be $8.78 \mathrm{GeV}$ and corresponding $\epsilon_{\delta}$ is 2.46. Therefore for such mass splitting, according to [80], the Sommerfeld enhancement can be negligible in the inert scalar models.

Moreover, Sommerfeld enhancement also affects the thermal freeze-out of the minimal DM as pointed out in $[47,48]$. Such enhancement is also expected in the case of inert scalar DM. But if the thermal freeze-out happens after the electroweak phase transition, one can introduce enough mass splitting so that $\epsilon_{\delta}>1$. In fact, $\delta=1.5 \mathrm{GeV}$ is compatible with the observed DM relic density of the universe with $m_{S}=3 \mathrm{TeV}$ for both doublet and quartet scalar DM. On the other hand, if freeze-out temperature, $T_{F}$ is larger than the critical temperature of electroweak phase transition, $T_{\mathrm{PT}}$, the thermal freeze-out takes place before the electroweak phase transition and there will not be any mass splitting to suppress the enhancement. Therefore thermal DM scenario of inert scalar DM will be different than that of the broken phase. But the value of the critical temperature of the electroweak phase transition depends on the model, order of the transition and its dynamics (see for example $[86,87])$. For this reason, we consider the range, $T_{\mathrm{PT}}=100-200 \mathrm{GeV}$ for the transition temperature. Now if $x_{f}=M_{\mathrm{DM}} / T_{F} \sim 20$, we see that for $m_{\mathrm{DM}}>4 \mathrm{TeV}$, freeze 


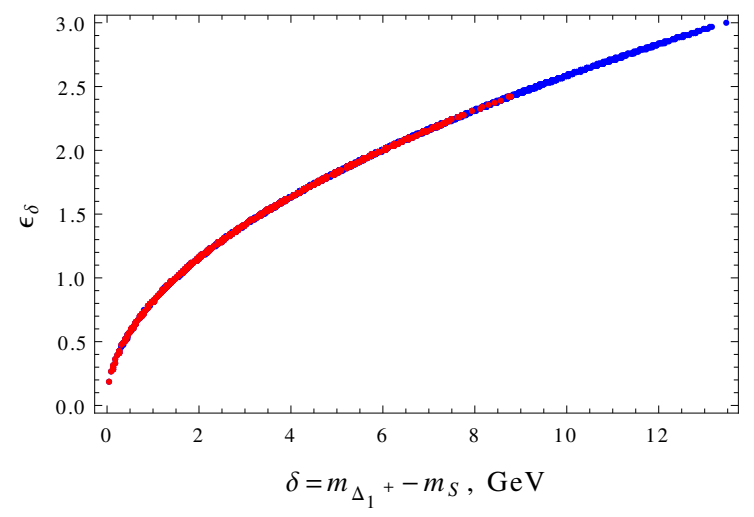

Figure 3. $\epsilon_{\delta}$ vs $\delta=m_{\Delta_{1}^{+}}-m_{S}$ for DM mass, $m_{S}=3000 \mathrm{GeV}$ in the quartet. Here, blue points are allowed by stability conditions on the scalar potential and perturbative limits on scalar couplings. Red points are allowed by the bounds on electroweak precision observables.

out takes place in the unbroken phase and will involve Sommerfeld enhanced annihilation cross-sections.

On the other hand, for $M_{\mathrm{DM}}<4 \mathrm{TeV}$, the DM freezes out in the broken phase. So one can introduce the enough mass splitting, $\delta \sim 1.5 \mathrm{GeV}$ between the DM and next to lightest charged state to suppress the enhancement in the annihilation cross sections.

For the inert scalar multiplets, apart from gauge interactions, the DM interacting via higgs exchange is also expected to have enhancement. In this case, the Yukawa potential $V_{\text {sc }}$ experienced by the DM is

$$
V_{\mathrm{sc}}(r)=\alpha_{\mathrm{sc}} \frac{e^{-m_{h} r}}{r} \text { with } \alpha_{\mathrm{sc}}=\frac{\lambda_{S}^{2}}{4 \pi} \frac{v^{2}}{m_{S}^{2}}
$$

For example, if $m_{S}=1 \mathrm{TeV}$ and $\lambda_{S}=\pi, \alpha_{\mathrm{sc}}=0.047$ so $\epsilon_{\phi}=2.6$ for the Higgs exchange, therefore the enhancement is generally not important for scalar interaction with the DM mass at $\mathrm{TeV}$ range.

In summary, although the $\mathrm{DM}$ with mass at $\mathrm{TeV}$ range in the inert scalar model is expected to have Sommerfeld enhancement in the gauge interactions and can have significantly enhanced indirect detection signal, there is a small common region of parameter space for doublet and quartet as seen from figure 2 (right) where one can have enough mass splitting to suppress the Sommerfeld enhancement in the inert scalar models and such mass splitting is compatible with the observed DM relic density. Therefore in the subsequent analysis, we only focus that small region of parameter space with benchmark point, $m_{S}=3 \mathrm{TeV}$ and $\delta=1.5 \mathrm{GeV}$ and have left the complete analysis of Sommerfeld enhancement in the inert quartet case for future work [90].

\subsubsection{Scalar coupling and LFV rates with scalar DM}

There is a correlation between the $\gamma$ coupling of the scalar sector and the rate of LFV processes when $R$ in eq. (2.1) is a real orthogonal matrix. As we can see from eq. (2.15) and eq. (2.17) that the smaller value of $\gamma$ leads to smaller value of the loop factor $\Lambda_{i}$ and thus neutrino mass. This in turn increases the Yukawa coupling, as in eq. (2.20), and 

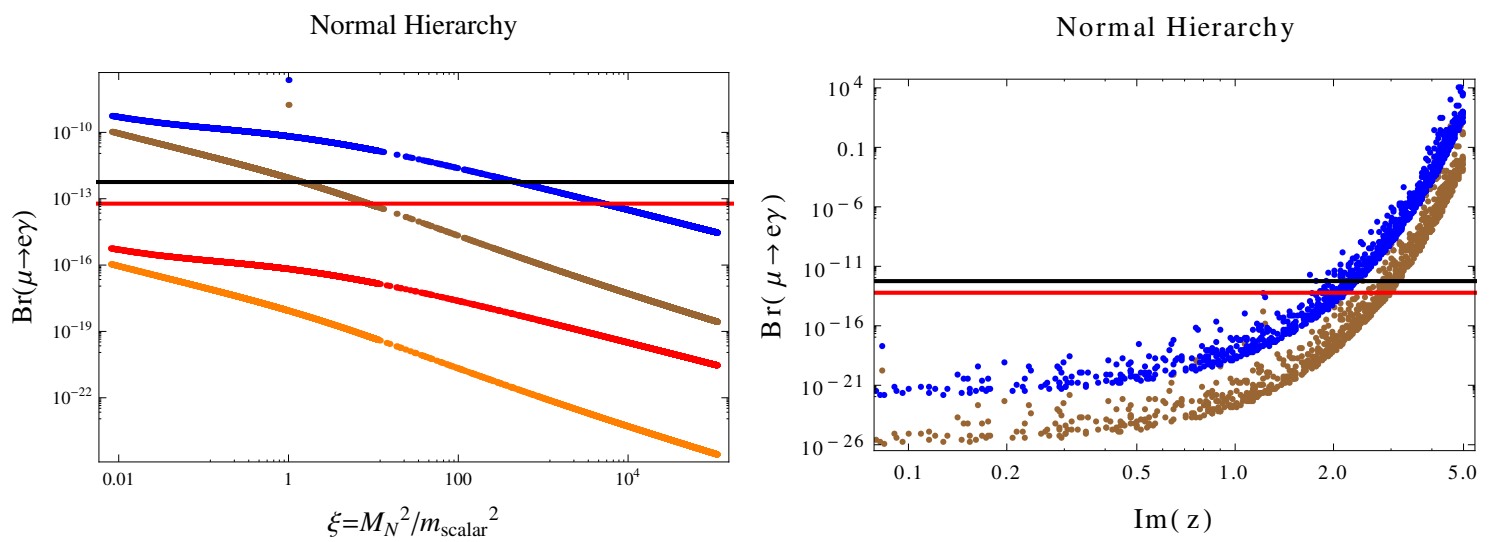

Figure 4. Left figure presents the dependence of the rate of LFV processes on the $\gamma$ when the $R$ is a real matrix. Here we have considered only $\operatorname{Br}(\mu \rightarrow e \gamma)$ for illustration. The brown and blue represents the rate in the doublet and quartet cases respectively for $\gamma=10^{-9}$. On the other hand, the orange and red points represents the rate in the doublet and quartet cases respectively for $\gamma=10^{-5}$. Right figure presents the correlation of the rate in doublet (brown points) and quartet (blue points) with imaginary part of the complex angle, $\operatorname{Im}(\mathrm{z})$, when we consider complex $R$ matrix. Here The scalar mass is fixed at $m_{\text {scalar }}=3000 \mathrm{GeV}$ and $\gamma=10^{-5}$. The black horizontal line is the current bound $5.7 \times 10^{-13}$ and red line is projected bound $6 \times 10^{-14}$.

becomes inconsistent with perturbativity bound eq. (2.21) when $\gamma$ is very small. On the other hand, large value of $\gamma$ implies larger separation in $m_{S}$ and $m_{A}$ and also in $m_{\Delta_{1}^{+}}$and $m_{\Delta_{2}^{+}}$, thus larger value of $\Lambda_{i}$ and in this case the value of Yukawa coupling is reduced. In figure 4 (left), We have illustrated this by comparing $\operatorname{Br}(\mu \rightarrow e \gamma)$ for $\gamma=10^{-9}$ and $10^{-5}$ respectively. We can see that for $\gamma=10^{-5}$, the rate has become out of reach for current and future experiments. Therefore in the case of real $R$ matrix, $\gamma \sim O\left(10^{-9}\right)$ leads to appreciable LFV rates. However we have seen in section 4.1.2 that as one would require, $\gamma \gtrsim 10^{-5}$ to kinematically forbid the inelastic scattering of scalar $\mathrm{DM}$ with $\mathrm{O}(\mathrm{TeV})$ mass so considering only real $R$ will lead to negligible rates of LFV processes.

On the other hand, in the case of complex $R$, such correlation between $\gamma$ and the rates of LFV processes is not straightforward because the size of Yukawa coupling also depends on the imaginary part of the complex angles in $R$. For simplicity, we have added an imaginary part, $\operatorname{Im}(\mathrm{z})$, in three angles of $R$ and in figure 4 (right), we can see that, despite having $\gamma=10^{-5}, \operatorname{Br}(\mu \rightarrow e \gamma)$ become comparable to the current bound with increasing values of $\operatorname{Im}(\mathrm{z})$. Again perturbativity of the Yukawa coupling typically put upper bound on $\operatorname{Im}(\mathrm{z})$ of $O(3-5)$. Therefore, one can have viable scalar DM in both doublet and quartet models where $\xi>1$ with appreciable LFV rates by tuning the Imaginary part of complex angles in $R$.

\subsubsection{Viable parameter space}

The parameter space for the model consists of $\left\{M_{0}, \alpha, \beta, \gamma\right\}$ of the scalar sector and $\left\{M_{N(F)}, y_{i \alpha}\right\}$ of the fermionic sector. Here $M_{N}$ and $M_{F}$ are the masses of RH neutrino and real fermion triplet (as the components of the triplet are degenerate at tree level) respectively. 
The focus of this preliminary study is the comparison among different LFV rates in doublet and quartet model with scalar DM. At first, from figure 2, as an exemplary point, we have chosen the mass of scalar DM to be $m_{S}=3 \mathrm{TeV}$ with $\lambda_{S}=1.3$ in the $m_{S}-\lambda_{S}$ plane so that scalar DM is viable both in doublet and quartet model. Moreover, $\gamma$ is set to be $10^{-5}$ to be consistent with bounds from DM direct detection. As the components of the scalar multiplet are almost degenerate apart from the very small splitting induced by non zero $\gamma$. Therefore we set the average mass of the scalar components at $m_{\text {scalar }}=3 \mathrm{TeV}$.

There are two sets of fermion mass range we have considered in our analysis. For the comparison of LFV rates with the variation of fermion masses both in doublet and quartet model, we have evaluate them in two sets, namely, i) where $\xi=M_{N(F)}^{2} / m_{\text {scalar }}^{2}<1$ so that the scalar component ceases to be the DM and ii) where $\xi>1$ where the scalar component is the DM. We have varied the masses of $\mathrm{RH}$ neutrinos and the fermion triplet within the range, $M_{N(F)} \in(270 \mathrm{GeV}, 30 \mathrm{TeV})$ which encompasses both sets mentioned above. $270 \mathrm{GeV}$ is taken as the lower limit of fermion mass as triplet fermion is excluded up to that mass in collider searches. Also such range is considered to see how the LFV rates vary with the mass of the fermion in addition to the DM aspects of inert scalar model.

We have used the experimental values of low energy neutrino parameters, $U_{\text {PMNS }}$, $\Delta m_{\text {solar }}^{2}$ and $\Delta_{\text {atm }}^{2}$ as the input in eq. (2.1) for Yukawa couplings. For both normal and inverted hierarchies, we could only vary the lowest neutrino mass, $m_{\nu_{1}}$, the Dirac phase, $\delta$ and Majorana phases, $\alpha_{\nu}, \beta_{\nu}$ and three complex angles, $z_{1}, z_{2}, z_{3}$ of, $R$. In our numerical analysis, as an simplification, the lowest neutrino mass is set to $m_{\nu}=1 \mathrm{meV}, \delta \in[0,2 \pi]$, $\alpha=\beta=0$ and common imaginary part in $z_{i}=\theta_{i}+i \operatorname{Im}\left(z_{i}\right), \operatorname{Im}(\mathrm{z})$ with the range $(0,5)$.

Summarizing, our input parameters in the numerical scans are $\left\{M_{0}, \alpha, \beta, \gamma, M_{N}=\right.$ $\left.M_{F}=\tilde{M}, m_{\nu_{1}}, \delta, \alpha_{\nu}, \beta_{\nu}, \theta_{1}, \theta_{2}, \theta_{3}, \operatorname{Im}(z)\right\}$ satisfying all the constraints mentioned above. Therefore, we can compare the LFV rates in both models for common viable point in the parameter space.

\subsection{LFV processes}

In the inert scalar models with scalar DM in the high mass regime, there is no direct correlation between the Yukawa couplings and DM properties. Also we have seen that the real matrix $R$ and $\gamma \gtrsim 10^{-5}$ (scalar DM direct detection constraint) give rise to small Yukawa couplings which in turn lead to LFV rates beyond the reach of current and future experiments as seen in figure 4 (left). But the size of the Yukawa coupling can be enhanced by varying the imaginary part of complex angles of $R$ without substantially affecting the phenomenology of the scalar DM and despite having $\gamma \gtrsim 10^{-5}$, we can easily obtain the LFV rates within the experimental range.

So first we have compared the rates of $\mu \rightarrow e \gamma, \mu \rightarrow e e \bar{e}$ and $\mu-e$ conversion rate with $\gamma=10^{-5}$ and the real $R$ matrix by varying the fermion masses for the doublet and quartet models. Then we vary $\operatorname{Im}(\mathrm{z})$ within its constrained limits and determine the region allowed by current and future bounds on the rates of these three LFV processes for both doublet and quartet cases. Also when $\xi>1$, we have our scalar dark matter in both doublet and quartet models. 
Normal Hierarchy

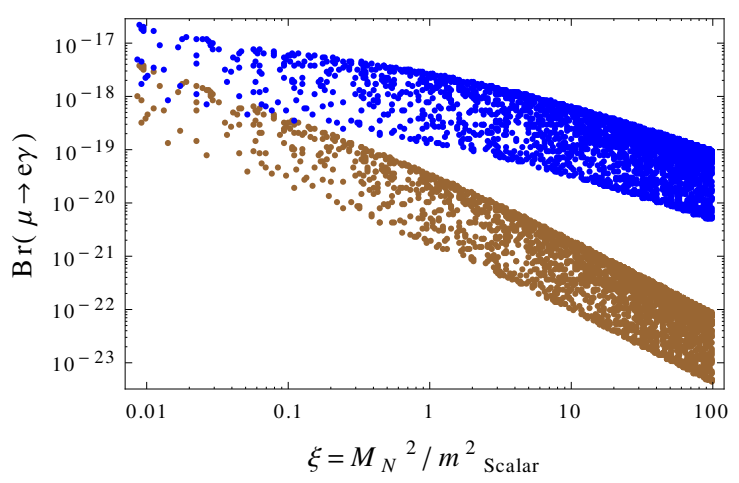

Inverted Hierarchy

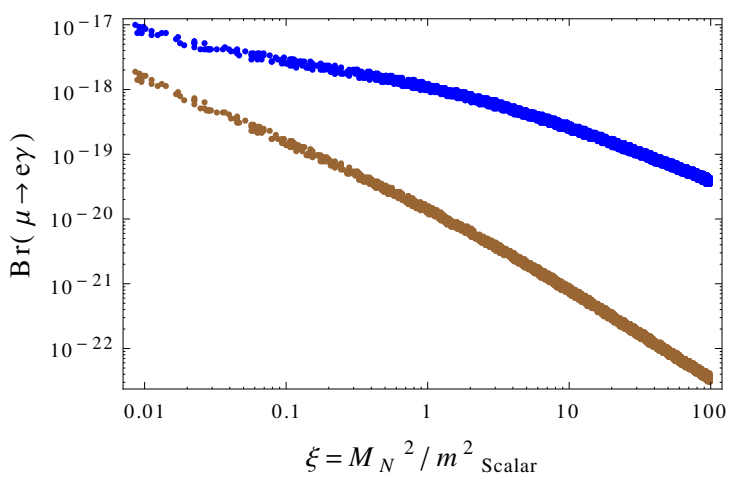

Figure 5. Correlation between $\xi=M_{N(F)}^{2} / m_{\text {scalar }}^{2}$ and $\operatorname{Br}(\mu \rightarrow e \gamma)$ for doublet (brown points) and quartet (blue points) with normal (left figure) and inverted (right figure) hierarchy for light neutrino mass. Here we have taken $M_{N(F)}$ to be degenerate, random Dirac phase $\delta$ and random real matrix $R$. Also we have set Majorana phases $\alpha_{\nu}$ and $\beta_{\nu}$ to be zero in this case. The scalar mass is fixed at $m_{\text {scalar }}=3000 \mathrm{GeV}$. Also $\gamma=10^{-5}$ and light neutrino mass, $m_{\nu_{1}}=1 \mathrm{meV}$.

\subsection{1 $\quad \operatorname{Br}(\mu \rightarrow e \gamma)$}

Due to the excellent bound put by the MEG collaboration [33, 34], $\mu \rightarrow e \gamma$ is one of the most well studied LFV processes. Figure 5 shows the comparison of this process between the doublet (brown points) and the quartet (blue points) scalar. We can see that the quartet contribution to $\mu \rightarrow e \gamma$ is larger than that of the doublet. For the same parameter point, in the quartet case, additional charged and neutral scalar $\left(\Delta_{1}^{ \pm}, \Delta_{2}^{ \pm}, \Delta^{ \pm \pm}, S\right.$ and $\left.A\right)$ and fermion states $\left(F_{i}^{0}\right.$ and $\left.F_{i}^{ \pm}\right)$enter in the loop compared to single charged scalar $\left(C^{ \pm}\right)$ and neutral fermion state $\left(N_{i}\right)$ in the doublet case and as the contributions of extra states are additive, the rate has increased in the quartet case than that of the doublet. From figure 5 we can see that $\operatorname{Br}(\nu \rightarrow e \gamma)$ is larger for the quartet than the doublet for both $\xi<1$ and $\xi>1$ (where the doublet and quartet scalars are the DM).

\subsection{2 $\operatorname{Br}(\mu \rightarrow e e \bar{e})$}

In $\mu \rightarrow e e \bar{e}$, the dominant contributions are coming from $\gamma$-penguin and Box diagrams. The Higgs penguin diagram is suppressed by the small electron Yukawa coupling. The $\mathrm{Z}$ penguin contribution is small because of the cancellation that takes place between $C_{24}$ and $B_{1}$ terms in eq. (3.12) and also between the same terms in eq. (3.15) when $m_{\sigma_{1}}=m_{\sigma_{2}}$. Moreover, similar cancellation takes place between the first two lines and third line of eq. (3.16) due to the specific relations among the couplings in front of the vertices. Therefore $\mathrm{Z}$ penguin contribution is also small in $\mu \rightarrow e e \bar{e}$ for both inert doublet and quartet case. Also note that the $\mathrm{Z}$ contribution in the quartet case is relatively bigger than that in the doublet because in the quartet $m_{\sigma_{1}}$ and $m_{\sigma_{2}}$ are not exactly equal when $\sigma_{1} \neq \sigma_{2}$. Hence one receives larger Z-penguin contribution in the quartet compared to the doublet. Still this contribution is numerically not significant if we compare it with $\gamma$ penguin diagram or box diagram contributions. From figure 6 , we can see that $\operatorname{Br}(\mu \rightarrow e e \bar{e})$ is larger for quartet (blue points) compared to the doublet (brown points) for both $\xi<1$ and $\xi>1$ cases. 
Normal Hierarchy

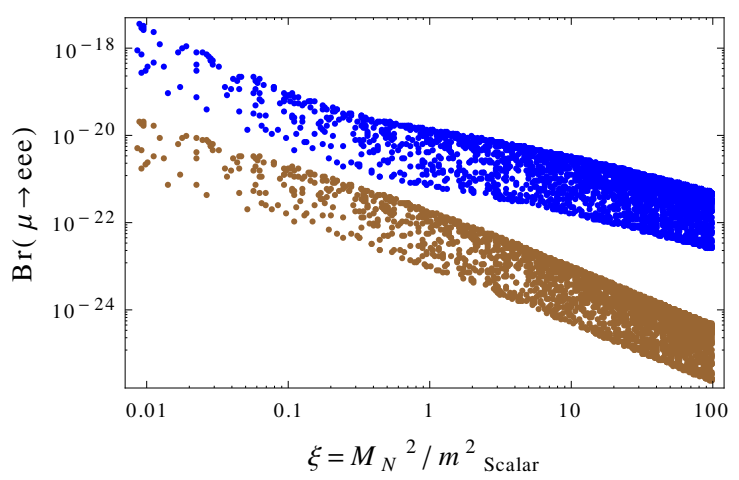

Inverted Hierarchy

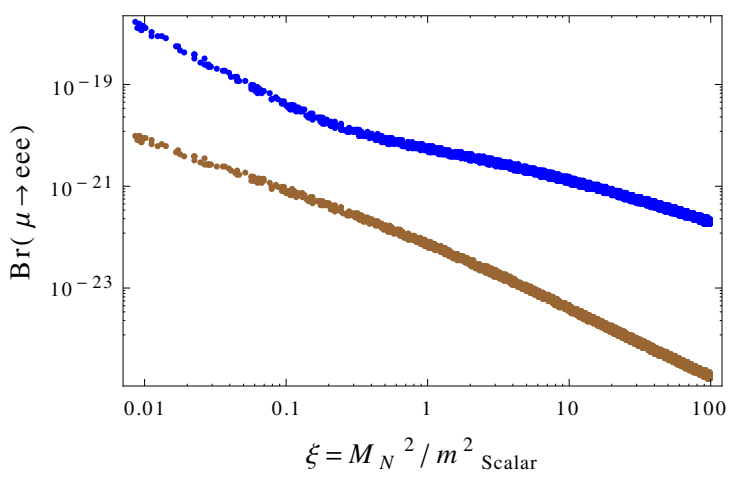

Figure 6. Correlation between $\xi=M_{N(F)}^{2} / m_{\text {scalar }}^{2}$ and $\operatorname{Br}(\mu \rightarrow e e \bar{e})$ for doublet (brown points) and quartet (blue points) with normal (left figure) and inverted (right figure) hierarchy for light neutrino mass. Here we have taken same input parameters as in $\operatorname{Br}(\mu \rightarrow e \gamma)$.

\subsection{3 $\mu-e$ conversion rate}

Another prominent LFV process currently under investigation is the $\mu-e$ conversion in nuclei. Here we have calculated the $\mu-e$ conversion rate for $\mathrm{Ti}$ and $\mathrm{Au}$ nuclei in the inert model with doublet and quartet. From figure 7 , we can see that the $\mu-e$ conversion rate is larger for the quartet (blue points) compared to the doublet (brown points). The dip occurs in the doublet contribution at $\xi=1$ because at that value, the dipole contribution $A_{D}^{\text {doublet }}$ and the non-dipole contribution $A_{N D}^{\text {doublet }}$ are equal as they are coming from single $\gamma$ penguin diagram involving charged scalar $C^{ \pm}$and neutral fermion $N_{i}$ and eq. (3.26) indicates that the effective coupling is zero for doublet at that point. On the other hand, for quartet case $A_{D}^{\text {quartet }}$ and $A_{N D}^{\text {quartet }}$ at $\xi=1$ are different because more than one charged scalar contribute to the $\gamma$ penguin diagrams. Again we can see from figure 7 that the conversion rate is larger for the quartet than that for the doublet for both $\xi<1$ and $\xi>1$ cases. We have not included the figure for $\mu-e$ conversion rate in Au nuclei as it is similar to figure 7 .

\subsubsection{LFV rates in the doublet and quartet}

As expected, the LFV rates seen in figure 5, 6 and 7 are very small for real $R$ and $\gamma=10^{-5}$. The rates will reduce even more if we increase $\gamma$. Still the rates are larger for the quartet compared to the doublet for $\xi<1$ and $\xi>1$ case where scalar is treated as the DM candidate. Now we increase the value of $\operatorname{Im}(z)$ and calculate the LFV rates with increasing values of $\tilde{M}$.

From figure 8, we can see that LFV rates in the quartet are more constrained than those in the doublet for common parameter space satisfying all the restrictions of section 4.1. The allowed regions on $\xi-\operatorname{Im}(\mathrm{z})$ plane are reduced further for both doublet and quartet models if one imposes the sensitivity of future lepton flavor violating experiments. The case for inverted hierarchy shows similar pattern so we have only presented results regarding normal hierarchy. 
Normal Hierarchy

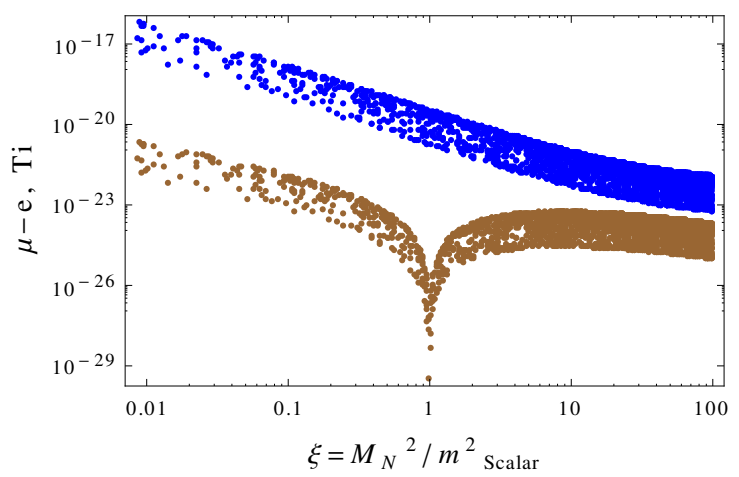

Inverted Hierarchy

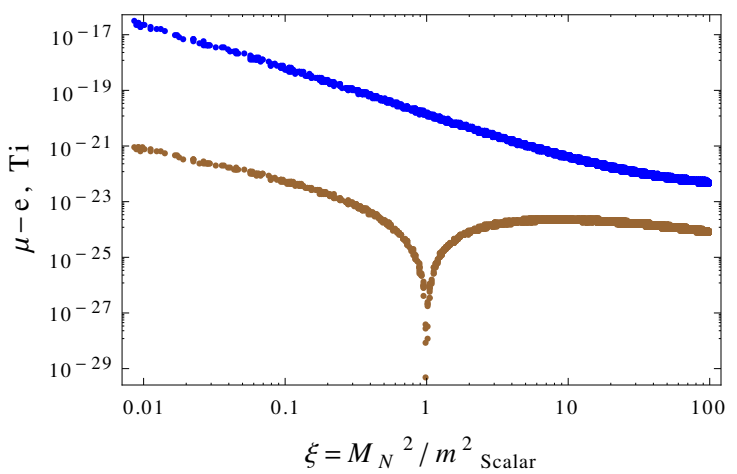

Figure 7. Correlation between $\xi=M_{N(F)}^{2} / m_{\text {scalar }}^{2}$ and $\mu-e$ conversion rate for Ti nucleus. for doublet (brown points) and quartet (blue points) with normal (left figure) and inverted (right figure) hierarchy for light neutrino mass. Here we have taken same input parameters as in $\operatorname{Br}(\mu \rightarrow e \gamma)$.
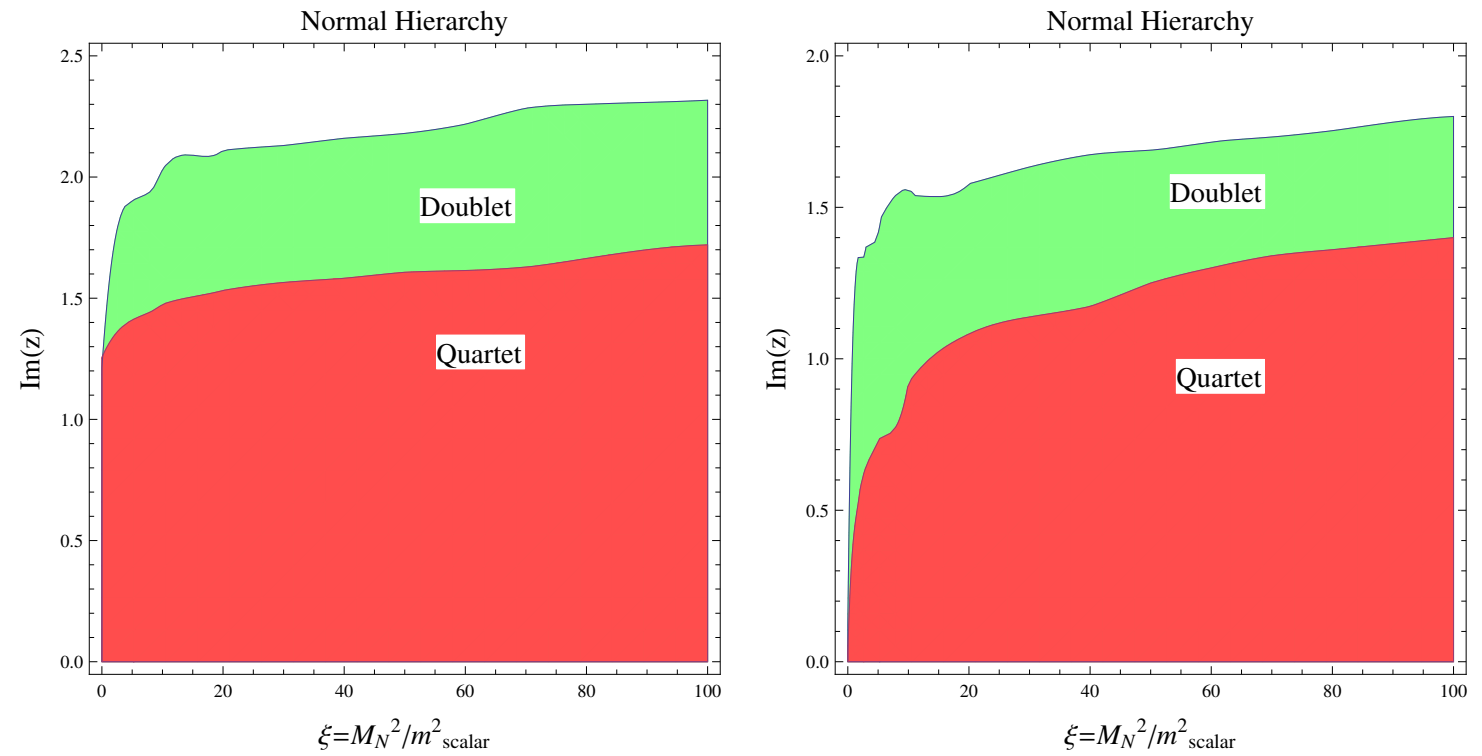

Figure 8. The $\xi-\operatorname{Im}(\mathrm{z})$ plane for degenerate $M_{N(F)}$, random Dirac phase $\delta$, zero Majorana phases $\alpha_{\nu}=\beta_{\nu}=0$ and light neutrino mass, $m_{\nu_{1}}=1 \mathrm{meV}$. The scalar mass is $m_{\text {scalar }}=3000 \mathrm{GeV}$ with $\gamma=10^{-5}$. In (left), the current bounds are imposed: $\operatorname{Br}(\mu \rightarrow e \gamma) \lesssim 5.9 \times 10^{-13}, \operatorname{Br}(\mu \rightarrow e e \bar{e}) \lesssim$ $1 \times 10^{-12}$ and $\mu-e$ conversion rate for $\mathrm{Ti} \lesssim 4.3 \times 10^{-12}$. In (right), the future sensitivity are considered: $\operatorname{Br}(\mu \rightarrow e \gamma) \lesssim 6.4 \times 10^{-14}, \operatorname{Br}(\mu \rightarrow e e \bar{e}) \lesssim 1 \times 10^{-16}$ and $\mu-e$ conversion rate for $\mathrm{Ti} \lesssim 10^{-18}$.

\section{Conclusions}

The scotogenic model is a well studied neutrino mass model and lepton flavor violation is one of its important phenomenological aspects. In this study we present the comparison among different LFV processes in the inert doublet and the quartet model, taking into account the current experimental limits and future sensitivity. There are two possible dark 
matter candidates in the inert scalar models: scalar and fermionic DM. In this study we have considered scalar DM and evaluated LFV rates for common parameter space subjected to collider bounds, DM constraints for doublet and quartet model and low-energy neutrino parameters. Our results are summarized as follows

- $\operatorname{Br}(\mu \rightarrow e \gamma), \operatorname{Br}(\mu \rightarrow e e \bar{e})$ and $\mu-e$ conversion rates in nuclei in the quartet model are larger than those in the doublet model for the same parameter space as seen from figure 5,6 and 7. In the case of higher scalar representation more particles enter into the loops and their contributions are additive in the LFV processes. Therefore we can have larger rates of different LFV processes compared to the lower scalar representation. From figure 8, we can see that, LFV processes in higher scalar representation are more constrained by the current and near-future experiments. In addition, this phenomenological result is complementary to the appearance of low scale Landau pole for higher representations $[22,88,89]$.

- There is no significant deviation from figure 5-8 for non-degenerate right handed neutrinos and real fermion triplets. In the case of large hierarchy, $m_{N_{3}} \gg m_{N_{1,2}}$, the dominant contribution comes from only the lightest generation.

We would like to emphasize here that the conclusion of our preliminary study is applicable to the inert scalar models where scalar DM is considered. But there is much room for an improved analysis. For example, in the case of fermionic DM, the DM constraints will be different and will have different viable parameter set for the LFV rate comparison. Also one needs to study the DM properties and viability of a common parameter space where $\xi \sim 1$. Therefore, further quantitative analysis of the fermionic DM aspects in the quartet model will be presented in a future publication [90]. Furthermore, similar analysis can be carried out for $\tau \rightarrow \mu \gamma, \tau \rightarrow e e \bar{e}, \tau \rightarrow \mu \mu \bar{\mu}$ in the inert scalar models to probe the flavor structure of the Yukawa sector and to have better constraints on the higher scalar representation in the light of experimental limits.

\section{Acknowledgments}

We would like to thank Avelino Vicente for stimulating discussion. T.A.C is grateful to Fernando Quevedo, Bobby Acharya and the HECAP section of ICTP for the support and the hospitality where the initial part of this work has been carried out. We are also indebted to the Referee for the constructive report, for which, the result and presentation of our study has been substantially improved.

\section{A Scalar masses}

\section{A.1 Inert doublet}

The mass spectrum for the inert doublet in our parametrization eq. (2.2) is,

$$
m_{S}^{2}=M_{0}^{2}+\frac{1}{2}\left(\alpha+\frac{1}{4} \beta+\gamma\right) v^{2}
$$




$$
\begin{aligned}
& m_{A}^{2}=M_{0}^{2}+\frac{1}{2}\left(\alpha+\frac{1}{4} \beta-\gamma\right) v^{2} \\
& m_{C}^{2}=M_{0}^{2}+\frac{1}{2}\left(\alpha+\frac{1}{4} \beta\right) v^{2}
\end{aligned}
$$

\section{A.2 Inert quartet}

In the inert quartet case, the $\gamma$ term, apart from splitting $S$ and $A$, also mixes two single charged components of the quartet. According to eq. (2.9), the mass matrix for single charged fields in $\left(\Delta^{+}, \Delta^{\prime}\right)$ basis is

$$
M_{+}^{2}=\left(\begin{array}{cc}
M_{0}^{2}+\frac{1}{2}\left(\alpha-\frac{1}{4} \beta\right) v^{2} & \frac{\sqrt{3}}{2} \gamma v^{2} \\
\frac{\sqrt{3}}{2} \gamma v^{2} & M_{0}^{2}+\frac{1}{2}\left(\alpha+\frac{3}{4} \beta\right) v^{2}
\end{array}\right)
$$

Diagonalizing the mass matrix, we have mass eigenstates for single charged fields, $\Delta_{1}^{+}=$ $\Delta^{+} \cos \theta+\Delta^{\prime} \sin \theta, \Delta_{2}^{+}=-\Delta^{+} \sin \theta+\Delta^{\prime}+\cos \theta$ with $\tan 2 \theta=-\frac{2 \sqrt{3} \gamma}{\beta}$.

Therefore the mass spectrum of the quartet is

$$
\begin{aligned}
m_{S(A)}^{2} & =M_{0}^{2}+\frac{1}{2}\left(\alpha+\frac{1}{4} \beta \mp 2 \gamma\right) v^{2} \\
m_{\Delta^{++}}^{2} & =M_{0}^{2}+\frac{1}{2}\left(\alpha-\frac{3}{4} \beta\right) v^{2} \\
m_{\Delta_{1}^{+}\left(\Delta_{2}^{+}\right)}^{2} & =M_{0}^{2}+\frac{1}{2}\left(\alpha+\frac{1}{2} \beta \mp \frac{1}{2} \sqrt{\beta^{2}+12 \gamma^{2}}\right) v^{2}
\end{aligned}
$$

Because of the mixing between two single charged states, the mass relation is

$$
m_{S}^{2}+m_{A}^{2}=m_{\Delta_{1}^{+}}^{2}+m_{\Delta_{2}^{+}}^{2}
$$

\section{B Loop functions}

The loop functions relevant for the dipole and non-dipole form factors from $\mu e \gamma$ vertex are

$$
\begin{aligned}
F^{(n)}(x) & =\frac{1-6 x+3 x^{2}+2 x^{3}-6 x^{2} \ln x}{6(1-x)^{4}} \\
F^{(c)}(x) & =\frac{2+3 x-6 x^{2}+x^{3}+6 x \ln x}{6(1-x)^{4}} \\
G^{(n)}(x) & =\frac{2-9 x+18 x^{2}-11 x^{3}+6 x^{3} \ln x}{6(1-x)^{4}} \\
G^{(c)}(x) & =\frac{16-45 x+36 x^{2}-7 x^{3}+6(2-3 x) \ln x}{6(1-x)^{4}}
\end{aligned}
$$

In the following we collect the Passarino-Veltman loop functions.

$$
B_{1}\left(m_{1}, m_{2}\right)=-\frac{1}{2}-\frac{m_{1}^{4}-m_{2}^{4}+2 m_{1}^{4} \ln \frac{m_{2}^{2}}{m_{1}^{2}}}{4\left(m_{1}^{2}-m_{2}^{2}\right)^{2}}+\frac{1}{2} \ln \frac{m_{2}^{2}}{\mu^{2}}
$$




$$
\begin{aligned}
C_{0}\left(m_{1}, m_{2}, m_{3}\right)= & \frac{m_{2}^{2}\left(m_{1}^{2}-m_{3}^{2}\right) \ln \frac{m_{2}^{2}}{m_{1}^{2}}-\left(m_{1}^{2}-m_{2}^{2}\right) m_{3}^{2} \ln \frac{m_{3}^{2}}{m_{1}^{2}}}{\left(m_{1}^{2}-m_{2}^{2}\right)\left(m_{1}^{2}-m_{3}^{2}\right)\left(m_{2}^{2}-m_{3}^{2}\right)} \\
C_{24}\left(m_{1}, m_{2}, m_{3}\right)= & \frac{1}{8\left(m_{1}^{2}-m_{2}^{2}\right)\left(m_{1}^{2}-m_{3}^{2}\right)\left(m_{2}^{2}-m_{3}^{2}\right)}\left[-2\left(m_{1}^{2}+m_{2}^{2}\right) m_{3}^{4} \ln \frac{m_{3}^{2}}{m_{1}^{2}}-\left(m_{3}^{2}-m_{1}^{2}\right)\right. \\
& \left.\left(2 m_{2}^{4} \ln \frac{m_{2}^{2}}{m_{1}^{2}}+\left(m_{1}^{2}-m_{2}^{2}\right)\left(m_{2}^{2}-m_{3}^{2}\right)\left(2 \ln \frac{m_{1}^{2}}{\mu^{2}}-3\right)\right)\right] \\
\tilde{D}_{0}\left(m_{1}, m_{2}, m_{3}, m_{4}\right)= & \frac{m_{2}^{4} \ln \frac{m_{2}^{2}}{m_{1}^{2}}}{\left(m_{2}^{2}-m_{1}^{2}\right)\left(m_{2}^{2}-m_{3}^{2}\right)\left(m_{2}^{2}-m_{4}^{2}\right)}-\frac{m_{3}^{4} \ln \frac{m_{3}^{2}}{m_{1}^{2}}}{\left(m_{3}^{2}-m_{1}^{2}\right)\left(m_{3}^{2}-m_{2}^{2}\right)\left(m_{3}^{2}-m_{4}^{2}\right)} \\
D_{0}\left(m_{1}, m_{2}, m_{3}, m_{4}\right)= & \frac{m_{2}^{2} \frac{m_{1}^{2}}{m_{1}^{2}}}{\left(m_{2}^{2}-m_{1}^{2}\right)\left(m_{2}^{2}-m_{2}^{2}-m_{3}^{2}\right)\left(m_{2}^{2}-m_{4}^{2}\right)}-\frac{m_{3}^{2} \ln \frac{m_{3}^{2}}{m_{1}^{2}}}{\left(m_{3}^{2}-m_{1}^{2}\right)\left(m_{3}^{2}-m_{2}^{2}\right)\left(m_{3}^{2}-m_{4}^{2}\right)} \\
& -\frac{m_{4}^{2} \ln \frac{m_{4}^{2}}{m_{1}^{2}}}{\left(m_{4}^{2}-m_{1}^{2}\right)\left(m_{4}^{2}-m_{2}^{2}\right)\left(m_{4}^{2}-m_{3}^{2}\right)}
\end{aligned}
$$

\section{C $\quad \mu e \gamma$ vertex, $\mu e Z$ vertex and box diagrams}

\section{C.1 $\mu e \gamma$ vertex}

Here we present in figure 9 the Feynman diagrams of one-loop contributions of the doublet and quartet to the $\mu e \gamma$ vertex.
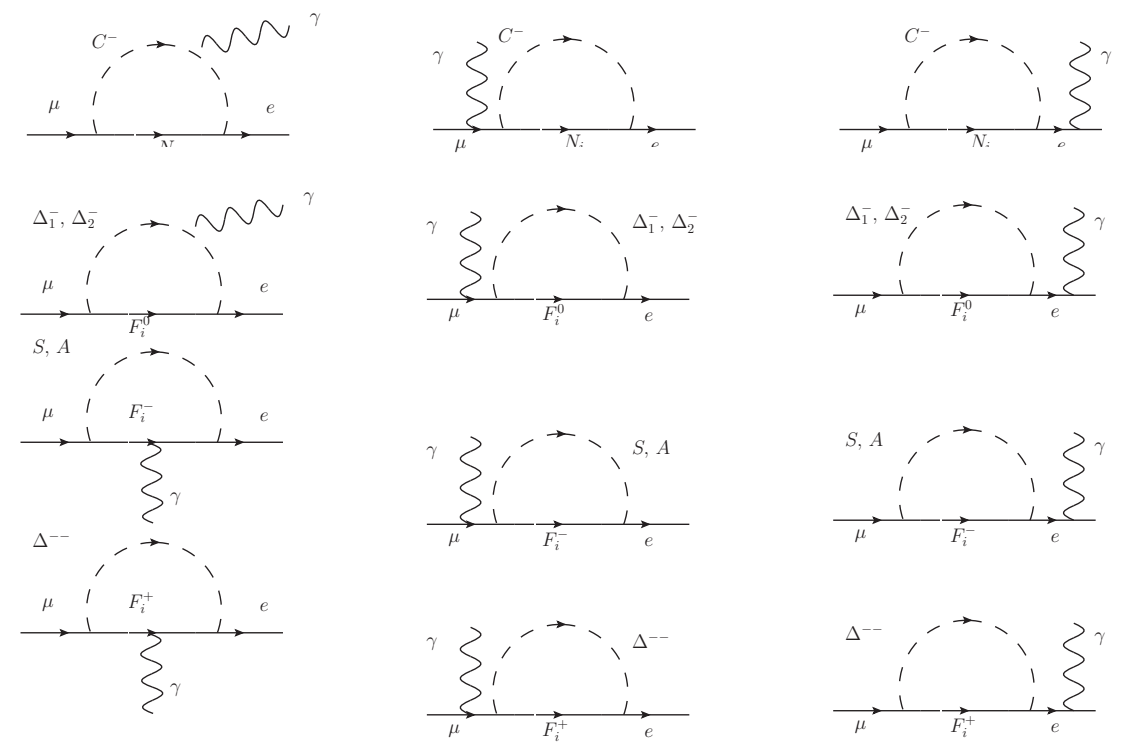

Figure 9. $\mu e \gamma$ vertex and the self energy diagrams of the external fermions for the doublet (first row) and the quartet cases (second to fourth rows). 


\section{C.2 $\mu e Z$ vertex}

We present in figure 10 the Feynman diagrams of one-loop contributions of the doublet and the quartet to the $\mu e Z$ vertex.
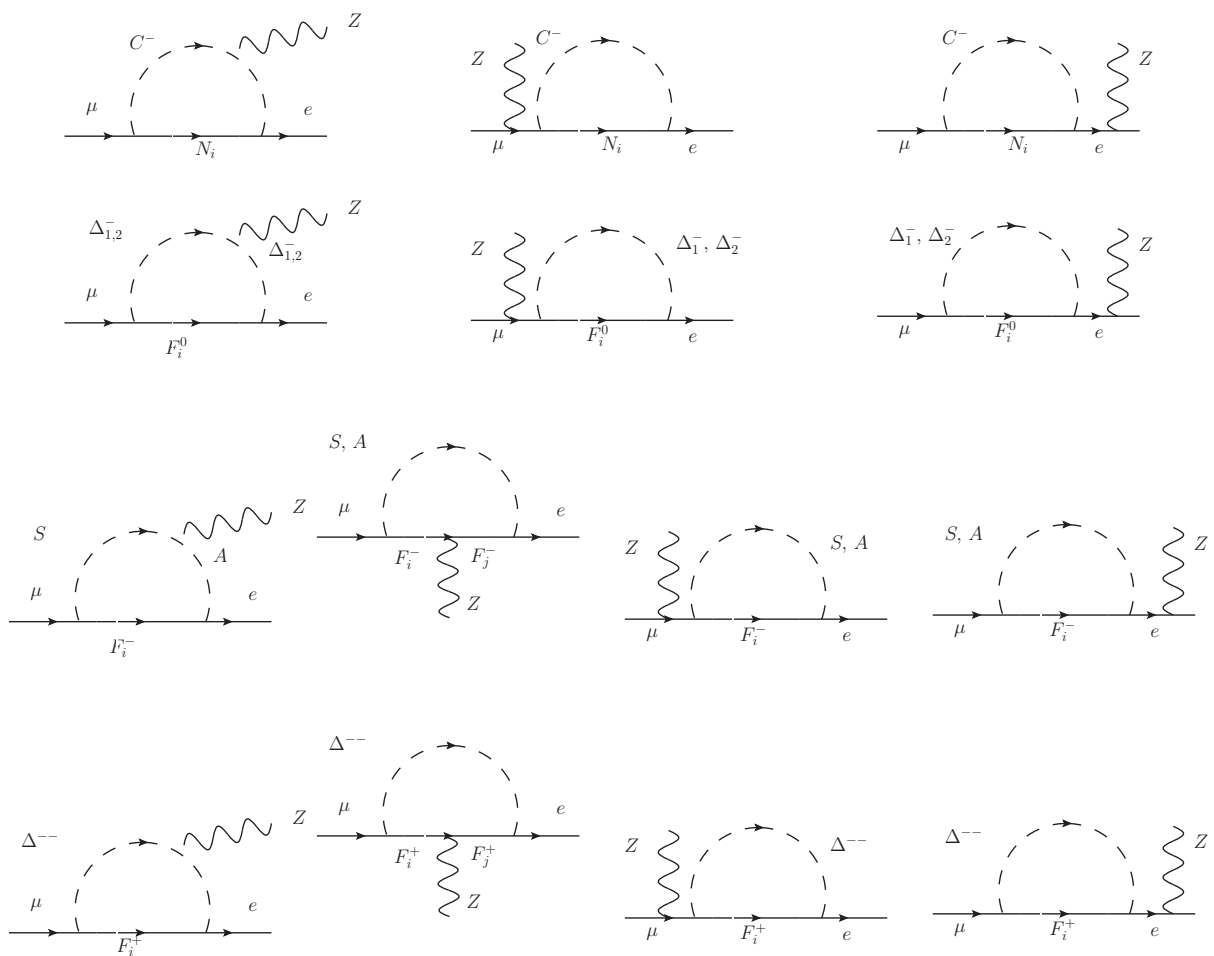

Figure 10. $\mu e Z$ vertex and the self energy diagrams of the external fermions for the doublet (first row) and the quartet cases (second to fourth rows). 


\section{C.3 Box diagrams}

The box diagrams for the doublet and the quartet cases are given in figure 11 .
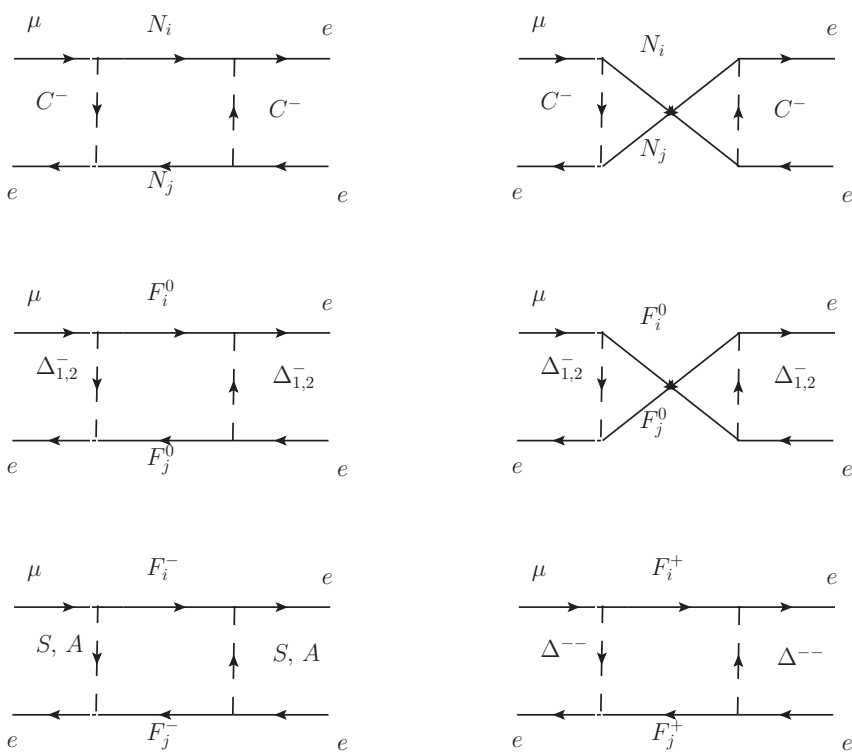

Figure 11. Box diagrams for the doublet (first row) and the quartet (second and third rows).

Open Access. This article is distributed under the terms of the Creative Commons Attribution License (CC-BY 4.0), which permits any use, distribution and reproduction in any medium, provided the original author(s) and source are credited.

\section{References}

[1] E. Ma, Verifiable radiative seesaw mechanism of neutrino mass and dark matter, Phys. Rev. D 73 (2006) 077301 [hep-ph/0601225] [INSPIRE].

[2] N.G. Deshpande and E. Ma, Pattern of Symmetry Breaking with Two Higgs Doublets, Phys. Rev. D 18 (1978) 2574 [INSPIRE].

[3] L. Lopez Honorez, E. Nezri, J.F. Oliver and M.H.G. Tytgat, The Inert Doublet Model: An Archetype for Dark Matter, JCAP 02 (2007) 028 [hep-ph/0612275] [INSPIRE].

[4] E.M. Dolle and S. Su, The Inert Dark Matter, Phys. Rev. D 80 (2009) 055012 [arXiv:0906.1609] [INSPIRE].

[5] L. Lopez Honorez and C.E. Yaguna, A new viable region of the inert doublet model, JCAP 01 (2011) 002 [arXiv:1011.1411] [InSPIRE].

[6] P. Agrawal, E.M. Dolle and C.A. Krenke, Signals of Inert Doublet Dark Matter in Neutrino Telescopes, Phys. Rev. D 79 (2009) 015015 [arXiv:0811.1798] [INSPIRE].

[7] S. Andreas, M.H.G. Tytgat and Q. Swillens, Neutrinos from Inert Doublet Dark Matter, JCAP 04 (2009) 004 [arXiv: 0901.1750] [INSPIRE]. 
[8] E. Nezri, M.H.G. Tytgat and G. Vertongen, $e^{+}$and $\bar{p}$ from inert doublet model dark matter, JCAP 04 (2009) 014 [arXiv:0901.2556] [INSPIRE].

[9] Q.-H. Cao, E. Ma and G. Rajasekaran, Observing the Dark Scalar Doublet and its Impact on the Standard-Model Higgs Boson at Colliders, Phys. Rev. D 76 (2007) 095011 [arXiv:0708.2939] [INSPIRE].

[10] H. Martinez, A. Melfo, F. Nesti and G. Senjanović, Three Extra Mirror or Sequential Families: A Case for Heavy Higgs and Inert Doublet, Phys. Rev. Lett. 106 (2011) 191802 [arXiv: 1101.3796] [INSPIRE].

[11] A. Melfo, M. Nemevšek, F. Nesti, G. Senjanović and Y. Zhang, Inert Doublet Dark Matter and Mirror/Extra Families after Xenon100, Phys. Rev. D 84 (2011) 034009 [arXiv: 1105.4611] [INSPIRE].

[12] T.A. Chowdhury, M. Nemevšek, G. Senjanović and Y. Zhang, Dark Matter as the Trigger of Strong Electroweak Phase Transition, JCAP 02 (2012) 029 [arXiv:1110.5334] [INSPIRE].

[13] D. Borah and J.M. Cline, Inert Doublet Dark Matter with Strong Electroweak Phase Transition, Phys. Rev. D 86 (2012) 055001 [arXiv:1204.4722] [INSPIRE].

[14] G. Gil, P. Chankowski and M. Krawczyk, Inert Dark Matter and Strong Electroweak Phase Transition, Phys. Lett. B 717 (2012) 396 [arXiv:1207.0084] [INSPIRE].

[15] J.M. Cline and K. Kainulainen, Improved Electroweak Phase Transition with Subdominant Inert Doublet Dark Matter, Phys. Rev. D 87 (2013) 071701 [arXiv:1302.2614] [INSPIRE].

[16] A. Ahriche, G. Faisel, S.-Y. Ho, S. Nasri and J. Tandean, Effects of two inert scalar doublets on Higgs boson interactions and the electroweak phase transition, Phys. Rev. D 92 (2015) 035020 [arXiv: 1501.06605] [INSPIRE].

[17] E. Lundstrom, M. Gustafsson and J. Edsjo, The Inert Doublet Model and LEP II Limits, Phys. Rev. D 79 (2009) 035013 [arXiv:0810.3924] [INSPIRE].

[18] E. Dolle, X. Miao, S. Su and B. Thomas, Dilepton Signals in the Inert Doublet Model, Phys. Rev. D 81 (2010) 035003 [arXiv: 0909.3094] [INSPIRE].

[19] M. Gustafsson, S. Rydbeck, L. Lopez-Honorez and E. Lundstrom, Status of the Inert Doublet Model and the Role of multileptons at the LHC, Phys. Rev. D 86 (2012) 075019 [arXiv: 1206.6316] [INSPIRE].

[20] M. Aoki, S. Kanemura and H. Yokoya, Reconstruction of Inert Doublet Scalars at the International Linear Collider, Phys. Lett. B 725 (2013) 302 [arXiv:1303.6191] [InSPIRE].

[21] G. Bélanger, B. Dumont, A. Goudelis, B. Herrmann, S. Kraml and D. Sengupta, Dilepton constraints in the Inert Doublet Model from Run 1 of the LHC, Phys. Rev. D 91 (2015) 115011 [arXiv: 1503.07367] [INSPIRE].

[22] S.S. AbdusSalam and T.A. Chowdhury, Scalar Representations in the Light of Electroweak Phase Transition and Cold Dark Matter Phenomenology, JCAP 05 (2014) 026 [arXiv: 1310.8152] [INSPIRE].

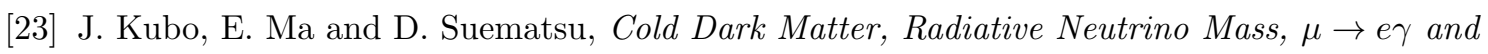
Neutrinoless Double Beta Decay, Phys. Lett. B 642 (2006) 18 [hep-ph/0604114] [INSPIRE].

[24] D. Aristizabal Sierra, J. Kubo, D. Restrepo, D. Suematsu and O. Zapata, Radiative seesaw: Warm dark matter, collider and lepton flavour violating signals, Phys. Rev. D 79 (2009) 013011 [arXiv: 0808.3340] [INSPIRE]. 
[25] D. Suematsu, T. Toma and T. Yoshida, Reconciliation of CDM abundance and $\mu \rightarrow e \gamma$ in a radiative seesaw model, Phys. Rev. D 79 (2009) 093004 [arXiv:0903.0287] [InSPIRE].

[26] A. Adulpravitchai, M. Lindner and A. Merle, Confronting Flavour Symmetries and extended Scalar Sectors with Lepton Flavour Violation Bounds, Phys. Rev. D 80 (2009) 055031 [arXiv: 0907.2147] [INSPIRE].

[27] T. Toma and A. Vicente, Lepton Flavor Violation in the Scotogenic Model, JHEP 01 (2014) 160 [arXiv: 1312.2840] [INSPIRE].

[28] A. Vicente and C.E. Yaguna, Probing the scotogenic model with lepton flavor violating processes, JHEP 02 (2015) 144 [arXiv:1412.2545] [INSPIRE].

[29] E. Ma and D. Suematsu, Fermion Triplet Dark Matter and Radiative Neutrino Mass, Mod. Phys. Lett. A 24 (2009) 583 [arXiv:0809.0942] [INSPIRE].

[30] S.S.C. Law and K.L. McDonald, A Class of Inert N-tuplet Models with Radiative Neutrino Mass and Dark Matter, JHEP 09 (2013) 092 [arXiv: 1305.6467] [INSPIRE].

[31] B. Ren, K. Tsumura and X.-G. He, A Higgs Quadruplet for Type III Seesaw and Implications for $\mu \rightarrow e \gamma$ and $\mu-e$ Conversion, Phys. Rev. D 84 (2011) 073004 [arXiv:1107.5879] [INSPIRE].

[32] A. Ahriche, K.L. McDonald, S. Nasri and T. Toma, A Model of Neutrino Mass and Dark Matter with an Accidental Symmetry, Phys. Lett. B 746 (2015) 430 [arXiv:1504.05755] [INSPIRE].

[33] MEG collaboration, J. Adam et al., New limit on the lepton-flavour violating decay $\mu^{+} \rightarrow e^{+} \gamma$, Phys. Rev. Lett. 107 (2011) 171801 [arXiv:1107.5547] [INSPIRE].

[34] MEG collaboration, J. Adam et al., New constraint on the existence of the $\mu^{+} \rightarrow e^{+} \gamma$ decay, Phys. Rev. Lett. 110 (2013) 201801 [arXiv:1303.0754] [INSPIRE].

[35] A.M. Baldini et al., MEG Upgrade Proposal, arXiv:1301.7225 [INSPIRE].

[36] SINDRUM collaboration, U. Bellgardt et al., Search for the Decay $\mu^{+} \rightarrow e^{+} e^{+} e^{-}$, Nucl. Phys. B 299 (1988) 1 [INSPIRE].

[37] A. Blondel et al., Research Proposal for an Experiment to Search for the Decay $\mu \rightarrow$ eee, arXiv:1301.6113 [INSPIRE].

[38] SINDRUM II collaboration, W.H. Bertl et al., A Search for muon to electron conversion in muonic gold, Eur. Phys. J. C 47 (2006) 337 [InSPIRE].

[39] SINDRUM II collaboration, C. Dohmen et al., Test of lepton flavor conservation in $\mu \rightarrow e$ conversion on titanium, Phys. Lett. B 317 (1993) 631 [INSPIRE].

[40] Mu2E collaboration, D. Glenzinski, The Mu2e Experiment at Fermilab, AIP Conf. Proc. 1222 (2010) 383 [INSPIRE].

[41] Mu2E collaboration, L. Bartoszek et al., Mu2e Technical Design Report, arXiv:1501.05241 [INSPIRE].

[42] DeeMe collaboration, H. Natori, DeeMe experiment - An experimental search for a mu-e conversion reaction at J-PARC MLF, Nucl. Phys. Proc. Suppl. 248-250 (2014) 52 [INSPIRE].

[43] COMET collaboration, A search for muon-to-electron conversion at J-PARC: The COMET experiment, PTEP 2013 (2013) 022C01.

[44] Y. Kuno, PRISM/PRIME, Nucl. Phys. Proc. Suppl. 149 (2005) 376 [InSPIRE]. 
[45] R.J. Barlow, The PRISM/PRIME project, Nucl. Phys. Proc. Suppl. 218 (2011) 44 [inSPIRE].

[46] M. Cirelli, N. Fornengo and A. Strumia, Minimal dark matter, Nucl. Phys. B 753 (2006) 178 [hep-ph/0512090] [INSPIRE].

[47] M. Cirelli, A. Strumia and M. Tamburini, Cosmology and Astrophysics of Minimal Dark Matter, Nucl. Phys. B 787 (2007) 152 [arXiv:0706.4071] [InSPIRE].

[48] M. Cirelli and A. Strumia, Minimal Dark Matter: Model and results, New J. Phys. 11 (2009) 105005 [arXiv: 0903.3381] [INSPIRE].

[49] J.A. Casas and A. Ibarra, Oscillating neutrinos and $\mu \rightarrow e, \gamma$, Nucl. Phys. B 618 (2001) 171 [hep-ph/0103065] [INSPIRE].

[50] J.A. Casas, J.M. Moreno, N. Rius, R. Ruiz de Austri and B. Zaldivar, Fair scans of the seesaw. Consequences for predictions on LFV processes, JHEP 03 (2011) 034 [arXiv: 1010.5751] [INSPIRE].

[51] J. Heeck, Seesaw parametrization for $n$ right-handed neutrinos, Phys. Rev. D 86 (2012) 093023 [arXiv: 1207.5521] [INSPIRE].

[52] T.P. Cheng and L.-F. Li, Nonconservation of Separate mu - Lepton and e - Lepton Numbers in Gauge Theories with v+a Currents, Phys. Rev. Lett. 38 (1977) 381 [INSPIRE].

[53] T.-P. Cheng and L.-F. Li, Muon Number Nonconservation Effects in a Gauge Theory with V A Currents and Heavy Neutral Leptons, Phys. Rev. D 16 (1977) 1425 [inSPIRE].

[54] T.P. Cheng and L.-F. Li, $\mu \rightarrow$ ey in Theories With Dirac and Majorana Neutrino Mass Terms, Phys. Rev. Lett. 45 (1980) 1908 [INSPIRE].

[55] E. Ma and A. Pramudita, Exact Formula for $(\mu \rightarrow e \gamma)$ Type Processes in the Standard Model, Phys. Rev. D 24 (1981) 1410 [InSPIRE].

[56] C.S. Lim and T. Inami, Lepton Flavor Nonconservation and the Mass Generation Mechanism for Neutrinos, Prog. Theor. Phys. 67 (1982) 1569 [InSPIRE].

[57] A. Ilakovac and A. Pilaftsis, Flavor violating charged lepton decays in seesaw-type models, Nucl. Phys. B 437 (1995) 491 [hep-ph/9403398] [INSPIRE].

[58] A. Blum and A. Merle, General Conditions for Lepton Flavour Violation at Tree- and 1-Loop Level, Phys. Rev. D 77 (2008) 076005 [arXiv:0709.3294] [INSPIRE].

[59] J. Hisano, T. Moroi, K. Tobe and M. Yamaguchi, Lepton flavor violation via right-handed neutrino Yukawa couplings in supersymmetric standard model, Phys. Rev. D 53 (1996) 2442 [hep-ph/9510309] [INSPIRE].

[60] E. Arganda and M.J. Herrero, Testing supersymmetry with lepton flavor violating tau and mu decays, Phys. Rev. D 73 (2006) 055003 [hep-ph/0510405] [INSPIRE].

[61] M.E. Krauss, W. Porod, F. Staub, A. Abada, A. Vicente and C. Weiland, Decoupling of heavy sneutrinos in low-scale seesaw models, Phys. Rev. D 90 (2014) 013008 [arXiv: 1312.5318] [INSPIRE].

[62] A. Abada, M.E. Krauss, W. Porod, F. Staub, A. Vicente and C. Weiland, Lepton flavor violation in low-scale seesaw models: SUSY and non-SUSY contributions, JHEP 11 (2014) 048 [arXiv: 1408.0138] [INSPIRE].

[63] E. Arganda and M.J. Herrero, Remark on the one-loop Z form factors for LFV Z-penguin diagrams in SUSY, arXiv:1403.6161 [INSPIRE]. 
[64] R. Kitano, M. Koike and Y. Okada, Detailed calculation of lepton flavor violating muon electron conversion rate for various nuclei, Phys. Rev. D 66 (2002) 096002 [Erratum ibid. D 76 (2007) 059902] [hep-ph/0203110] [INSPIRE].

[65] E. Arganda, M.J. Herrero and A.M. Teixeira, $\mu-e$ conversion in nuclei within the CMSSM seesaw: Universality versus non-universality, JHEP 10 (2007) 104 [arXiv:0707.2955] [INSPIRE].

[66] A. Crivellin, M. Hoferichter and M. Procura, Improved predictions for $\mu \rightarrow e$ conversion in nuclei and Higgs-induced lepton flavor violation, Phys. Rev. D 89 (2014) 093024 [arXiv: 1404.7134] [INSPIRE].

[67] Particle Data Group collaboration, J. Beringer et al., Review of Particle Physics (RPP), Phys. Rev. D 86 (2012) 010001 [inSPIRE].

[68] ATLAS collaboration, Search for charginos nearly mass degenerate with the lightest neutralino based on a disappearing-track signature in pp collisions at $\sqrt{s}=8 \mathrm{TeV}$ with the ATLAS detector, Phys. Rev. D 88 (2013) 112006 [arXiv:1310.3675] [INSPIRE].

[69] M. Cirelli, F. Sala and M. Taoso, Wino-like Minimal Dark Matter and future colliders, JHEP 10 (2014) 033 [Erratum ibid. 1501 (2015) 041] [arXiv: 1407.7058] [INSPIRE].

[70] Planck collaboration, P.A.R. Ade et al., Planck 2013 results. XVI. Cosmological parameters, Astron. Astrophys. 571 (2014) A16 [arXiv:1303.5076] [INSPIRE].

[71] T. Hambye, F.S. Ling, L. Lopez Honorez and J. Rocher, Scalar Multiplet Dark Matter, JHEP 07 (2009) 090 [Erratum ibid. 1005 (2010) 066] [arXiv:0903.4010] [INSPIRE].

[72] A. Alloul, N.D. Christensen, C. Degrande, C. Duhr and B. Fuks, FeynRules 2.0 - A complete toolbox for tree-level phenomenology, Comput. Phys. Commun. 185 (2014) 2250 [arXiv: 1310.1921] [INSPIRE].

[73] G. Bélanger, F. Boudjema, A. Pukhov and A. Semenov, MicrOMEGAs $s_{3}$ : A program for calculating dark matter observables, Comput. Phys. Commun. 185 (2014) 960 [arXiv: 1305.0237] [INSPIRE].

[74] LUX collaboration, D.S. Akerib et al., First results from the LUX dark matter experiment at the Sanford Underground Research Facility, Phys. Rev. Lett. 112 (2014) 091303 [arXiv: 1310.8214] [INSPIRE].

[75] A. Sommerfeld, Diffraction and Retardation of Electrons, Ann. Phys. 11 (1931) 257.

[76] J. Hisano, S. Matsumoto and M.M. Nojiri, Explosive dark matter annihilation, Phys. Rev. Lett. 92 (2004) 031303 [hep-ph/0307216] [INSPIRE].

[77] J. Hisano, S. Matsumoto, M.M. Nojiri and O. Saito, Non-perturbative effect on dark matter annihilation and gamma ray signature from galactic center, Phys. Rev. D 71 (2005) 063528 [hep-ph/0412403] [INSPIRE].

[78] J. Hisano, S. Matsumoto, O. Saito and M. Senami, Heavy wino-like neutralino dark matter annihilation into antiparticles, Phys. Rev. D 73 (2006) 055004 [hep-ph/0511118] [INSPIRE].

[79] N. Arkani-Hamed, D.P. Finkbeiner, T.R. Slatyer and N. Weiner, A Theory of Dark Matter, Phys. Rev. D 79 (2009) 015014 [arXiv: 0810.0713] [INSPIRE].

[80] T.R. Slatyer, The Sommerfeld enhancement for dark matter with an excited state, JCAP 02 (2010) 028 [arXiv:0910.5713] [INSPIRE]. 
[81] J. Fan and M. Reece, In Wino Veritas? Indirect Searches Shed Light on Neutralino Dark Matter, JHEP 10 (2013) 124 [arXiv:1307.4400] [INSPIRE].

[82] T. Cohen, M. Lisanti, A. Pierce and T.R. Slatyer, Wino Dark Matter Under Siege, JCAP 10 (2013) 061 [arXiv:1307.4082] [INSPIRE].

[83] M. Cirelli, T. Hambye, P. Panci, F. Sala and M. Taoso, Gamma ray tests of Minimal Dark Matter, JCAP 10 (2015) 026 [arXiv:1507.05519] [INSPIRE].

[84] C. Garcia-Cely, A. Ibarra, A.S. Lamperstorfer and M.H.G. Tytgat, Gamma-rays from Heavy Minimal Dark Matter, JCAP 10 (2015) 058 [arXiv:1507.05536] [INSPIRE].

[85] M. Aoki, T. Toma and A. Vicente, Non-thermal Production of Minimal Dark Matter via Right-handed Neutrino Decay, JCAP 09 (2015) 063 [arXiv:1507.01591] [INSPIRE].

[86] M. Quirós, Finite temperature field theory and phase transitions, hep-ph/9901312 [INSPIRE].

[87] D.E. Morrissey and M.J. Ramsey-Musolf, Electroweak baryogenesis, New J. Phys. 14 (2012) 125003 [arXiv: 1206 . 2942] [INSPIRE].

[88] L. Di Luzio, R. Gröber, J.F. Kamenik and M. Nardecchia, Accidental matter at the LHC, JHEP 07 (2015) 074 [arXiv: 1504.00359] [INSPIRE].

[89] Y. Hamada, K. Kawana and K. Tsumura, Landau pole in the Standard Model with weakly interacting scalar fields, Phys. Lett. B 747 (2015) 238 [arXiv:1505.01721] [INSPIRE].

[90] T.A. Chowdhury and S. Nasri, in preparation. 